\title{
Zur Soziografie der Volksbegehren und Volksentscheide 1926 und 1929 in der Weimarer Republik
}

\author{
Dirk Hänisch
}

Volksbegehren und Volksentscheid als Elemente der Volksgesetzgebung waren in der Weimarer Reichsverfassung (WRV) verankert. Ein Volksbegehren mussten mindestens zehn Prozent der Stimmberechtigten befürworten, damit anschließend ein Volksentscheid durchgeführt wurde. Nur wenn sich an der Abstimmung die Mehrheit der Stimmberechtigten beteiligte, konnte in der Praxis ein Volksgesetz zustande kommen (Art. 75 WRV).

Die Weimarer Republik erlebte im Laufe ihrer Geschichte drei reichsweite Volksbegehren, davon führten zwei zu Volksentscheiden, die aber schließlich an der Mehrheitshürde scheiterten. Erfolglos blieb das Volksbegehren „Gegen den Panzerkreuzerbau“, das 1928 von der Kommunistischen Partei Deutschlands (KPD) initiiert wurde und mit weniger als drei Prozent nicht die erforderliche Mindestanzahl an Eintragungen erreichte ${ }^{1}$. Davor bzw. danach fanden 1926 und 1929 zwei Volksbegehren statt, die in reichsweite Abstimmungsverfahren mündeten, aber letztlich nicht die erforderliche Mehrheit der Stimmberechtigten des Reiches gewinnen konnten. Dies waren der Volksentscheid zur Enteignung der Fürstenvermögen und der Volksentscheid über den Entwurf eines „Gesetzes gegen die Versklavung des Deutschen Volkes", das die Reparationszahlungen im Young-Plan ablehnte.

Auch auf Landesebene gab es diese Form der politischen Partizipation. Insbesondere die KPD und die Nationalsozialistische Partei Deutschlands (NSDAP) instrumentalisierten in der Endphase der Weimarer Republik 1931 und 1932 Volksbegehren und Volksentscheide zur Erreichung ihrer jeweiligen politischen Ziele. So gab es (teilweise erfolgreiche) Versuche, die Landtage in Preußen, Anhalt, Thüringen, Braunschweig, Oldenburg und Lippe (1931), Bremen, Oldenburg und Sachsen (1932) aufzulösen. Obwohl die

1 Zu den Plebisziten in der Weimarer Republik O. Jung, Direkte Demokratie in der Weimarer Republik, Frankfurt a.M./New York 1989. Die reichsweiten Ergebnisse der Volksbegehren und Volksentscheide bei J.W. Falter/T. Lindenberger/S. Schumann, Wahlen und Abstimmungen in der Weimarer Republik, München 1986, S. 47. Vgl. auch Fn. 3. 
Flügelparteien sich dieser Form der Partizipation vermehrt bedienten, waren die Plebiszite keineswegs verantwortlich oder ursächlich für die Zerstörung oder Destabilisierung der Weimarer Demokratie.

Dieser Aufsatz hat die wahlstatistische Analyse der Ergebnisse der beiden reichsweiten Plebiszite - Volksbegehren und Volksentscheid - zur Enteignung der Fürstenvermögen (1926) und zur Ablehnung des Young-Plans (1929) zum Gegenstand ${ }^{2}$. Beide wurden jeweils von politisch konträren Kräften und Bündnissen initiiert und vorangetrieben. Mit den wahlstatistischen Methoden der historischen Wahlforschung und anhand eines umfangreichen Datenmaterials sollen zwei Fragen beantwortet werden: Welche parteipolitischen Wählergruppen unterstützten und mobilisierten bei beiden Plebisziten, und welches Sozialprofil wiesen ihre Unterstützer auf? Zusätzlich wird gefragt, ob es statistische Zusammenhänge des Ausgangs des Volksentscheides 1929 mit dem reichsweiten Aufstieg der NSDAP bei der Reichstagswahl im September 1930 gibt.

\section{Datenbasis und Methodik}

Den wahlstatistischen Auswertungen der beiden Volksbegehren und -entscheide liegen zwei vom Verfasser zusammengestellte Datensätze mit Wahl- und Sozialdaten der Kreise und Städte des Deutschen Reiches zugrunde. Es handelt sich um die in der Reihe Statistik des Deutschen Reichs ${ }^{3}$ veröffentlichten Ergebnisse der beiden Plebiszite sowie um die Ergebnisse der Volks- und Berufszählung auf Stadt- und Landkreisebene von $1925^{4}$. Durch umfangreiche und zeitaufwendige Recherchen in zahlreichen Archiven sowie durch das Studium einer Vielzahl zeitgenössischer Zeitungen

2 Der Wortlaut der beiden zur Abstimmung stehenden Gesetzentwürfe zur Fürstenenteignung und gegen den Young-Plan ist in der Statistik des Deutschen Reichs, Bd 332 (Berlin 1926), S. 3 und Bd. 372/III (Berlin 1931), S. 35 abgedruckt.

3 Die endgültigen Ergebnisse der beiden Plebiszite sind (nur auf Kreisebene) veröffentlicht worden in: Statistik (Fn. 2), Bd. 332 und 372/III. Die Vielzahl an zusätzlich durchgesehenen Zeitungen und Archivantworten kann aus Platzgründen hier nicht aufgeführt werden.

4 Aufgenommen wurden die berufliche Gliederung der Bevölkerung nach Wirtschaftsabteilungen und sozialer Stellung sowie die konfessionelle Zugehörigkeit der Kreise einschließlich der kreiszugehörigen Städte und Gemeinden ab 10.000 Einwohnern. Diese Daten finden sich ebenfalls in der Statistik (Fn. 2), Bd. 403-405 (Berlin 1927-29). Die konfessionelle Gliederung wurde Bd. 401/I (Berlin 1930) entnommen. 
wurden darüber hinaus Abstimmungsergebnisse für größere kreiszugehörige Städte ab 10.000 Einwohner ermittelt und damit die Datenbasis erweitert und verfeinert, so dass schließlich für das Plebiszit 1926 insgesamt 1.274 Auswertungseinheiten (Land- und Stadtkreise, 211 kreiszugehörige Städte ab 10.000 Einwohnern sowie die 20 Verwaltungsbezirke von Berlin) wahlstatistisch auswertbar sind. Beim Volksbegehren und dem anschließenden Volksentscheid gegen den Young-Plan von 1929 ergab die Presseüberlieferung ein etwas lückenhafteres Bild, so dass schließlich Daten von 1.118 Stadt- und Landkreisen (davon 97 kreiszugehörige Städte ab 10.000 Einwohner) zur Auswertung bereit stehen. Die beiden Datensätze enthalten außerdem die Wahlergebnisse der zeitlich vorangegangenen Reichstagswahlen vom Dezember 1924 bzw. Mai 19285. Aus Vergleichsgründen wurden zusätzlich zum Plebiszit 1929 auch die Ergebnisse der Reichstagswahl vom September 1930 aufgenommen. Beide Datensätze sind diachronisch organisiert, d.h. die Auswertungseinheiten sind im Zeitverlauf zwischen 1924 und 1926 bzw. 1925 und 1930 vergleichbar und geographisch relativ stabil. Die zwischen 1928 und 1929 zahlreichen regionalen Gebietsreformen, insbesondere im rheinisch-westfälischen Industriegebiet ${ }^{6}$, wurden im Datensatz entsprechend berücksichtigt ${ }^{7}$.

Die hier vorliegenden Daten sind Aggregatdaten, also Daten über Kollektive und nicht über Individuen. Damit sind unweigerlich methodologische Fragen und Probleme verbunden, die in der Fachliteratur unter dem Stichwort

5 Die Wahlergebnisse sind ausgewiesen für Kreise und kreiszugehörige Städte und Gemeinden ab 2000 Einwohner in der Statistik (Fn. 2): Dezember 1924 im Bd. 315 (1925), Mai 1928 im Bd. 372 (1930) und September 1930 im Bd. 382 (1932; alle Berlin).

6 Eine vergleichende kartografische Darstellung der massiven Gebietsreformen 1926 und insb. 1929 findet sich bei $H$. Ehlgötz, Die Umgemeindungen in Westdeutschland, in: Bauwirtschaft und Baurecht 1929, S. 161 ff., 165 ff. Instruktiv auch $H$. Hoebink, Mehr Raum - mehr Macht. Preußische Kommunalpolitik und Raumplanung im rheinisch-westfälischen Industriegebiet 1900-1933, Essen 1990.

7 Das erfolgte in der Regel durch Zusammenlegung zu einem neuen Aggregat, sobald festgelegte Populationsschwankungen überschritten wurden. Um Großaggregate zu vermeiden, wurden Stadtteilergebnisse aus lokalen Akten für die eingemeindeten Städte und Gemeinden ermittelt und entsprechend disaggregiert. Es handelt sich um Alt-Gelsenkirchen, Buer und Horst (Gelsenkirchen), Alt-Oberhausen, Sterkrade und Osterfeld (Oberhausen), Alt-Duisburg, Hamborn, Duisburg-Süd (Duisburg), Barmen, Elberfeld, Vohwinkel, Cronenberg und Ronsdorf (Wuppertal), Alt-Solingen, Ohligs, Wald, Gräfrath, Höhscheid (Solingen) sowie um einige weitere Städte außerhalb des Ruhrgebiets. Mein Dank gilt in diesem Zusammenhang den Stadtarchiven in den genannten Städten. 
ökologische Fehlschlussproblematik diskutiert wurden und immer noch werden, d.h. der Schluss von der Aggregat- auf die Individualebene ist nur unter bestimmten Umständen und Voraussetzungen zulässig ${ }^{8}$. Da aber für die Zeit der Weimarer Republik keine Individualdaten herangezogen werden können, weil die Umfrageforschung damals noch unbekannt war, ist eine (sorgfältige) Analyse von möglichst kleinräumigen Aggregatdaten heute die einzige Möglichkeit, einigermaßen zuverlässige Aussagen über das Wahl- bzw. Abstimmungsverhalten zu treffen.

Für Schlüsse von der Aggregat- auf die Individualebene eignen sich am ehesten statistische Inferenzverfahren, wie beispielsweise das ökologische Logit-Schätzverfahren des dänischen Politikwissenschaftlers S.R. Thomsen, das dieser in den 1980er Jahren entwickelt hat und häufig plausible Ergebnisse lieferte ${ }^{9}$, oder die ökologische Regression nach Goodman mit anschließenem Proportional-Fitting-Verfahren, das etwa von Falter und seinen Mitarbeitern im Rahmen der Erforschung des NSDAP-Aufstiegs eingesetzt wur$\mathrm{de}^{10}$. Dennoch muss stets betont werden, dass alle Verfahren bestenfalls

8 Die Diskussion über den ökologischen Fehlschluss ist bereits relativ alt und wurde durch W.S. Robinson, Ecological correlations and the behaviour of individuals, in: ASR 15 (1950), S. 351 ff. ausgelöst. Vgl. J.W. Falter, Hitlers Wähler, München 1991, S. $61 \mathrm{ff}$.

9 Vgl. S.R. Thomsen, Danish Elections 1920-1979. A Logit Approach to Ecological Analysis and Inference, Aarhus 1987.

10 L. Goodman, Ecological Regressions and Behavior of Individuals, in: American Sociological Review 18 (1953), S. 663-664. Das „Glättungsverfahren“ wie z.B. das Proportional Fitting nach Deming/Stephen, das die errechneten negativen Übergangswahrscheinlichkeiten wieder in den positiven Bereich ,zurückholt“, führt bei Wahlpaaren in der Regel sehr häufig immer dann zu unrealistischen Schätzungen, wenn die Übergangstabellen zwei Wahlpaare mit einem ähnlichen Parteienangebot abbilden. In solchen Fällen scheint das Thomsen-Verfahren realistischere Schätzwerte zu produzieren. Vgl. auch die Ausführungen bei D. Hänisch, Die österreichischen NSDAP-Wähler, Wien/Köln/Weimar 1998, S. 137 ff. Durch das Glättungsverfahren ist zu erklären, warum die auf diese Weise errechneten Haltequoten der Weimarer Parteien bei allen Wahlpaaren sehr moderat ausfallen, vgl. beispielsweise dazu die Werte in Tabelle 2 (obere Hälfte) bei J.W. Falter. The Social Bases of Political Cleavages in the Weimar Republic, 1919-1933, in: L.E. Jones/J. Retallack (Hrsg.), Elections, Mass Politics, and Social Change in Modern Germany, New York u.a. 1992, S. 371-397; neuerdings auch bei J.W.Falter, Political Cleavages in the Weimar Republic and the Rise of National Socialism, in: European Political Science 13 (2014), S. 106-116 (110). Das Deming-Stephen-Verfahren wird erläutert in: J.-B. Lohmöller/H. Bömermann, Ökologische Inferenz. Eine anwenderorientierte Einführung, Berlin 1993, S. 165 ff. 
„holzschnittartige“ Annäherungen an tatsächliche Größenordnungen bieten ${ }^{11}$. Ihre Schätzziffern beinhalten stets die Gefahr, fehlerbehaftet und ungenau zu sein. Da es aber mangels Umfragedaten keine anderen Quellen als Aggregatdaten gibt, um Wählerwanderungen und soziale Zusammensetzungen $\mathrm{zu}$ schätzen, sind diese Verfahren bisher so gut wie alternativlos.

Wir haben uns an diesem Punkt entschieden, auf das bei Wählerwanderungen gut bewährte Logit-Schätzverfahren von Thomsen zu verzichten und die klassische ökologische Regression nach Goodman mit anschließenden Proportional-Fitting zu verwenden ${ }^{12}$. In Dutzenden von Modellen und Berechnungen lieferte die ökologische Regression in den vorliegenden Fällen, wo Übergangswahrscheinlichkeiten zwischen Wahlen und Volksentscheiden zu schätzen waren anders als das Thomsen-Verfahren deutlich plausiblere und nachvollziehbarere Werte. Den Grund dafür sehen wir in dem Fehlen der von Thomsen geforderten „,neutralen Kategorie“ der Nichtwähler bei den beiden hier vorliegenden Volksabstimmungen als grundlegende Bezugsgröße in seinem Logit-Schätzverfahren. Diese Annahme wird aber nicht eingehalten, denn bekanntlich repräsentierten die ausgewiesenen Nichtwähler beim Volksentscheid nicht jene Nichtwähler im klassischen Sinne, wie wir sie etwa von Reichstagswahlen her kennen, sondern sie enthalten faktisch auch solche Personengruppen, die den Volksentscheid bewusst ablehnten, etwa durch das (ausdrücklich empfohlene) Fernbleiben von der Abstimmungsurne.

Mit den hier präsentierten wahlstatistischen Auswertungen wird gewissermaßen wahlhistorisches Neuland betreten, denn die Volksentscheide in der Weimarer Republik sind nie Gegenstand der empirischen historischen Wahlforschung gewesen und wurden bei den Analysen zu den Wählerbewe-

11 Auch G. King, A Solution to the Ecological Inference Problem: Reconstructing Individual Behavior from Aggregate Data, Princeton/New Jersey 1997 hat eine statistische Lösung des Inferenzproblems unter Einbeziehung der Methods of Bounds von Duncan und Davis für Vierfeldertabellen zu entwickeln versucht. Sie ist aber trotz des vielversprechenden Buchtitels auch noch nicht die ultimative Lösung des Problems. So steht insbesondere bei Mehrfeldertabellen ein befriedigender Lösungsansatz aus. Ambühl kommt bei seiner Expertise der vorhandenen Verfahren zur Schätzung von Wählerwanderungen aus Aggregatdaten zu dem recht harschen Ergebnis, dass selbst bei Erfüllung der Modellanforderungen sämtliche vorliegende Verfahren $\mathrm{zu}$ eher spekulativen Ergebnissen der Wählerwanderungsanalyse führen $(M$. Ambühl, Methoden zur Rekonstruktion von Wählerströmen aus Aggregatdaten, Neuchatel 2003, S. 87.

12 Die Berechnungen erfolgten mit dem von Lohmöller geschriebenen Programm PLS 1.8. Vgl. dazu Lohmöller/Bömermann, Inferenz (Fn. 10). 
gungen in der zweiten Hälfte der Weimarer Republik kaum berücksichtigt ${ }^{13}$. Die meisten Betrachtungen und Ausführungen im Rahmen etwa von Monografien begnügten sich mit Wahlkreisvergleichen, globalen Saldierungen und der Analyse von Einzelergebnissen ${ }^{14}$. Dieser Aufsatz versucht nun, diese Forschungslücke zu schließen und die politischen und sozialen Kräfte, die die beiden Volksentscheide stützten, näher zu bestimmen.

\section{Volksbegehren und Volksentscheid im März bzw. Juni 1926 zur Fürs- tenenteignung}

Die Initiative zu diesem Plebiszit ging ursprünglich von der KPD aus, später nach der Jahreswende 1925/26 schloss sich nach anfänglichem Zögern und auf Druck der Parteibasis die Führung der Sozialdemokratischen Partei Deutschlands (SPD) an. Ziel war die entschädigungslose Enteignung der Vermögen der Fürstenhäuser, die mit der Novemberrevolution ihre politische Macht verloren hatten. Auch Teile der Deutschen Demokratischen Partei (DDP) befürworteten ebenso wie einige Verbände und zahlreiche Intellektuelle die Initiative, aus der bald eine breite Bewegung wurde. Obwohl KPD und SPD das Vorhaben unterstützten, gab es vor Ort keine gemeinsam durchgeführten Veranstaltungen oder Aktionen beider Parteien. Dennoch können Volksbegehren und Volksentscheid 1926 als das einzige gemeinsam getragene politische Vorhaben von SPD und KPD in der Weimarer Republik klassifiziert werden. Zentrum, Bayerische Volkspartei (BVP) und Deutsche Volkspartei (DVP) lehnten das Plebiszit ab und sprachen sich gegen eine Beteiligung an Volksbegehren und Volksentscheid aus. Deutschnationale Volkspartei (DNVP) und völkisch-nationalsozialistischen Kräfte etwa in Ge-

13 Ausnahme: Falter u.a., Wahlen (Fn. 1), S. 203. Teilergebnisse wurden später übernommen in ders., Wähler (Fn. 8), S. 362. Dort wurde der Volksentscheid über die Fürstenenteignung 1926 als Indikator für ,linke politische Tradition“" in die Analyse eingeführt. Beide Volksentscheide waren aber nicht Gegenstand eingehender Auswertungen. Die umfangreichen wahlstatistischen Auswertungen des Teams um Falter, dem der Autor im Zeitraum von 1984-1989 angehörte, berücksichtigten die Volksentscheide nicht weiter.

14 Das gilt insb. für U. Schüren, Der Volksentscheid zur Fürstenenteignung 1926, Düsseldorf 1978; F.C. West, A Crisis of the Weimar Republic: A Study of the German Referendum of 20 June 1926, Diss. phil. Univ. of California 1970 (1985); O. Jung, Volksgesetzgebung. Die „Weimarer Erfahrungen“ aus dem Fall der Vermögensauseinandersetzungen zwischen Freistaaten und ehemaligen Fürsten, Bd. 2, Hamburg 1990. 
stalt der Listenverbindung Nationalsozialistische Freiheitsbewegung (NSFB) waren scharfe Gegner und sahen im Volksentscheid den Versuch von, wie es hieß, bolschewistischer Seite, den Einstieg in zukünftige Enteignungen von Vermögen und Eigentum vorzubereiten ${ }^{15}$.

Die Diskussionen wurden zusätzlich durch den überraschend guten Ausgang des Volksbegehrens befördert, denn über 12,5 Millionen Wahlberechtigte trugen sich vom 4. bis 17. März 1926 in die ausliegenden Stimmenlisten ein, was einem Anteil von 31,5 Prozent entsprach und damit nicht nur erheblich über dem geforderten Quorum von zehn Prozent lag, sondern auch den gemeinsamen Anteil von KPD und SPD (zusammen knapp 27,2 Prozent, DDP 4,9 Prozent) bei der vorangegangenen Reichstagswahl vom Dezember 1924 überflügelte ${ }^{16}$. In der Zentrumspartei, die in einigen Regionen wie etwa im westlichen und mittleren Ruhrgebiet über eine nennenswerte Arbeiterbasis verfügte, löste die Haltung der Parteiführung interne Diskussionen aus, auf die beispielsweise die zentrumsnahe Presse unmittelbar vor dem Abstimmungstag mit entsprechenden Anzeigen der Parteiführung reagierte, um den offiziellen Standpunkt der Partei gegenüber dem Parteipublikum zu unterstreichen ${ }^{17}$. Der Volksentscheid am 20. Juni 1926 fiel dann mit einem Zuspruch von knapp 36,4 Prozent (über 14,4 Millionen Ja-Stimmen) doch enttäuschender aus und scheiterte somit.

15 Die Haltung u. politischen Positionen der gesellschaftlichen Akteure sind ausführlich zusammengestellt bei Jung, Volksgesetzgebung (Fn. 14).

16 Alle Prozentuierungen der Wahl- sowie der Plebiszitergebnisse erfolgten, wie in der historischen Wahlforschung üblich, grundsätzlich auf die Zahl der Wahl- bzw. Stimmberechtigten, um den gesamten Wahlkörper entsprechend abzubilden. Ebenso wurden alle Korrelationen mit den Berechtigtenziffern gewichtet.

17 So am 19.6.1926 in der Essener Volkszeitung, dem führenden Zentrumsblatt in der Industriestadt. „Zentrumsanhänger in Essen-Stadt und -Land! Bei der Abstimmung über den Volksentscheid [...] bitten wir unsere Wähler, sich an die Richtlinien des Reichsparteivorstandes zu halten. Diese lauten: ,Der Reichsvorstand erwartet, daß die Zentrumsanhänger nicht für das Enteignungsgesetz stimmen'. Diese Parole läßt folgende Möglichkeiten zu: a) der Wähler kann zu Hause bleiben und sich an der Wahl überhaupt nicht beteiligen. b) Der Wähler beteiligt sich an der Wahl dadurch, daß er ungültig wählt, d.h. einen leeren Briefumschlag (ohne Wahlzettel) abgibt.“ 
a) Die regionale Herkunft der Befürworter von Volksbegehren und Volksentscheid

Wo lagen die regionalen Zentren der Befürworter des Volksbegehrens bzw. des Volksentscheids? Und wo wurde am erfolgreichsten gegenüber dem Volksbegehren mobilisiert? Antworten darauf liefern die beiden thematischen Karten 1 und 2. Alle Auswertungseinheiten (Kreise, Städte und Verwaltungsbezirke) wurden nach der Höhe ihrer Ja-Stimmenanteile in Quartile eingeteilt und ihre Zugehörigkeit in die Karteneinheiten entsprechend eingefärbt übertragen ${ }^{18}$. Das oberste (am dunkelsten eingefärbte) Quartil zeigt das

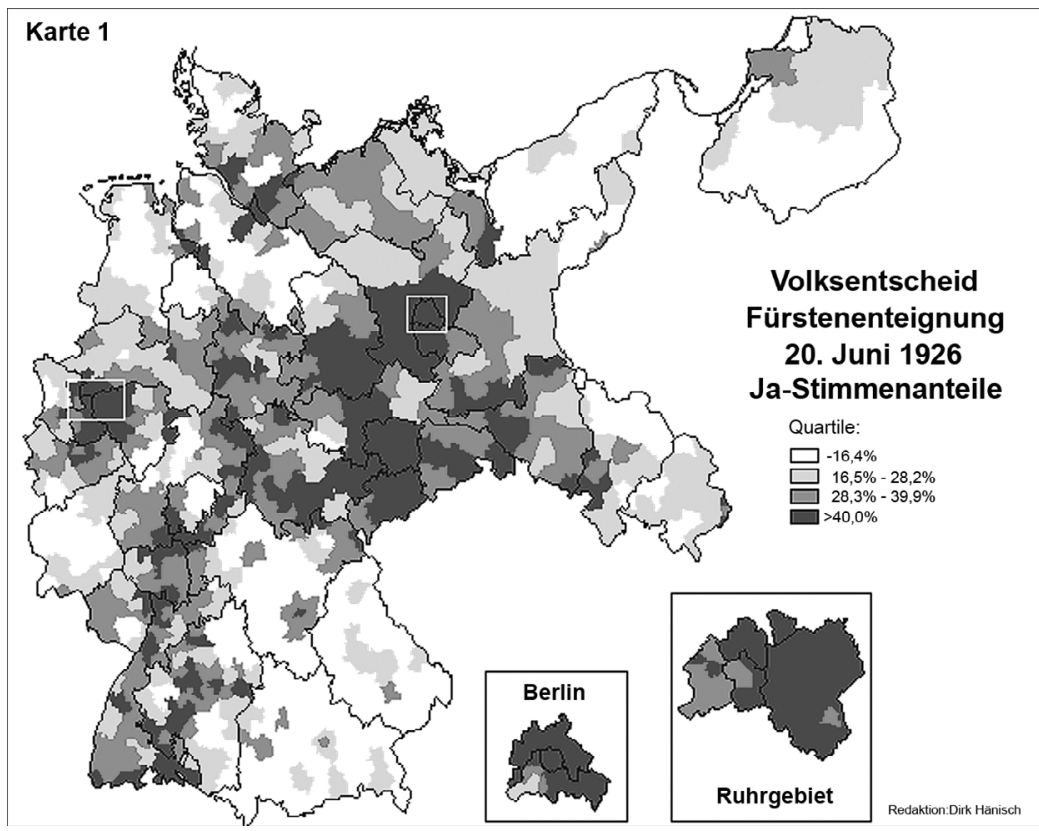

18 Vorlage für die reichsweite Karte war die Kartenbeilage aus Bd. 418 der Statistik (Fn. 2), Gebietsstand nach der Volks- und Berufszählung von 1925. Die kartografische Darstellung birgt zwangsläufig den Nachteil, dass die zahlreichen Stadtkreise wegen ihrer geringen Größe bei diesem Maßstab nicht dargestellt werden können, so dass sich die thematischen Karten auf Darstellung von etwa 900 Flächenkreisen beschränken. Die Daten der Stadtkreise wurden in die entsprechenden zugehörigen Landkreisflächen eingerechnet. Die Quartilsberechnung selbst erfolgte auf der Basis aller Land- und Stadtkreise einschließlich der Berliner Verwaltungsbezirke. In die hier dargestellten Karten wurden auch die Wahlkreisgrenzen, bei der Sonderkarte Berlin die Verwaltungsbezirksgrenzen und bei der Ruhrgebietskarte die Regierungsbezirksgrenzen abgetragen. 
Viertel mit den höchsten Ja-Anteilen an, die hellen Flächen zeigen die Diasporagebiete.

$\mathrm{Zu}$ den Diasporagebieten zählten der Osten Preußens mit Ausnahme von Brandenburg, insbesondere die Provinzen Ostpreußen und Pommern, Regionen also, die man landläufig eher als ,plattes Land“ bezeichnet. Hinzu kommen aber auch große Teile Bayerns, ferner das Gebiet des heutigen Niedersachsens, das nördliche Schleswig-Holstein und der katholische Westen bei Trier. Bereits in der zeitgenössischen Presse sowie in der Fachliteratur wurde auf die erschwerten Bedingungen vor allem im agrarischen Osten Deutschlands hingewiesen, wenn man dort als Befürworter der Volksabstimmung von seinem Recht zur Einzeichnung in die Listen beim Volksbegehren bzw. von seinem Stimmrecht beim Volksentscheid Gebrauch machen wollte ${ }^{19}$. Sozialer, wirtschaftlicher und politischer Druck in Klein- und Kleinstgemeinden führten dazu, dass in den dortigen Gegenden die Befürworter lieber zu Hause blieben, als sich öffentlich zu Volksbegehren und Volksentscheid $\mathrm{zu}$ bekennen $^{20}$. Die Hochburgen des Volksentscheids lagen in Sachsen, Brandenburg, Berlin, Thüringen und im Gebiet des heutigen Sachsen-Anhalt, im Ruhrgebiet sowie im Bergischen Land, in Teilen von Hessen, im westlichen Württemberg und in Nordbaden, alles Gebiete, die eher durch eine fortgeschrittene Industrialisierung gekennzeichnet waren.

Die zweite thematische Karte zeigt die Regionen an, wo beim Volksentscheid stärker oder schwächer gegenüber dem Volksbegehren mobilisiert werden konnte - definiert als Zuwachs der Ja-Stimmen gegenüber den Ein-

19 Beispiele von Behinderungen und Einschränkungen schildert Schüren, Volksentscheid (Fn. 14), S. 118 f. Formal konnte auch bei den Volksentscheiden mit Nein abgestimmt werden, die Gegner zogen es aber vor, sich erst gar nicht an der Abstimmung zu beteiligen. Daher war der Anteil von Dissidentenstimmen äußerst gering und kann hier wie im Folgenden vernachlässigt werden.

20 So schrieb die sozialdemokratische Pfälzische Post (Ludwigshafen) am 18. März 1926 zum Ausgang des Volksbegehrens: „Haben wir nicht auch in der Pfalz Beispiele genug, daß Bürgermeisterämter das Möglichste taten, um die Einzeichnung in die Listen $\mathrm{zu}$ erschweren oder gar ganz unmöglich zu machen? [...] Als erschwerendstes Moment aber kommt zu dem allen noch die eigenhändige Einzeichnung in die Listen, was einer öffentlichen Stimmabgabe gleichkommt." Und die SPDZeitung Volksblatt (Bochum) bemerkte dazu: ,Was soll man dazu sagen, wenn, wie in Pommern Gutsbesitzer das Amt des Wahlleiters übernehmen und den Arbeitern, die die Absicht haben, gegen die Fürsten zu stimmen, sofort zu entlassen drohen? Was ist das für eine Wahlfreiheit, wenn, wie in Demmin, Reichswehrsoldaten durch die Stadt marschieren und sämtliche Plakate abreißen, die für die Fürstenenteignung werben? Aus Ostpreußen, Pommern, Schlesien werden Terrorakte in Unzahl gemel$\operatorname{det}[\ldots] . “$ 
tragungen, bezogen auf die Stimmberechtigtenzahl. Das Mobilisierungspotenzial war in den meisten Hochburgenregionen des Volksbegehrens bereits nahezu ausgeschöpft, so etwa in der ,roten“ Hochburg Sachsen, wo es in einigen Kreisen sogar Rückgänge gab ${ }^{21}$. Nennenswerte Zuwächse kamen überwiegend in jenen Gebieten zustande, die zu den Diasporaregionen des Volksbegehrens gehörten.

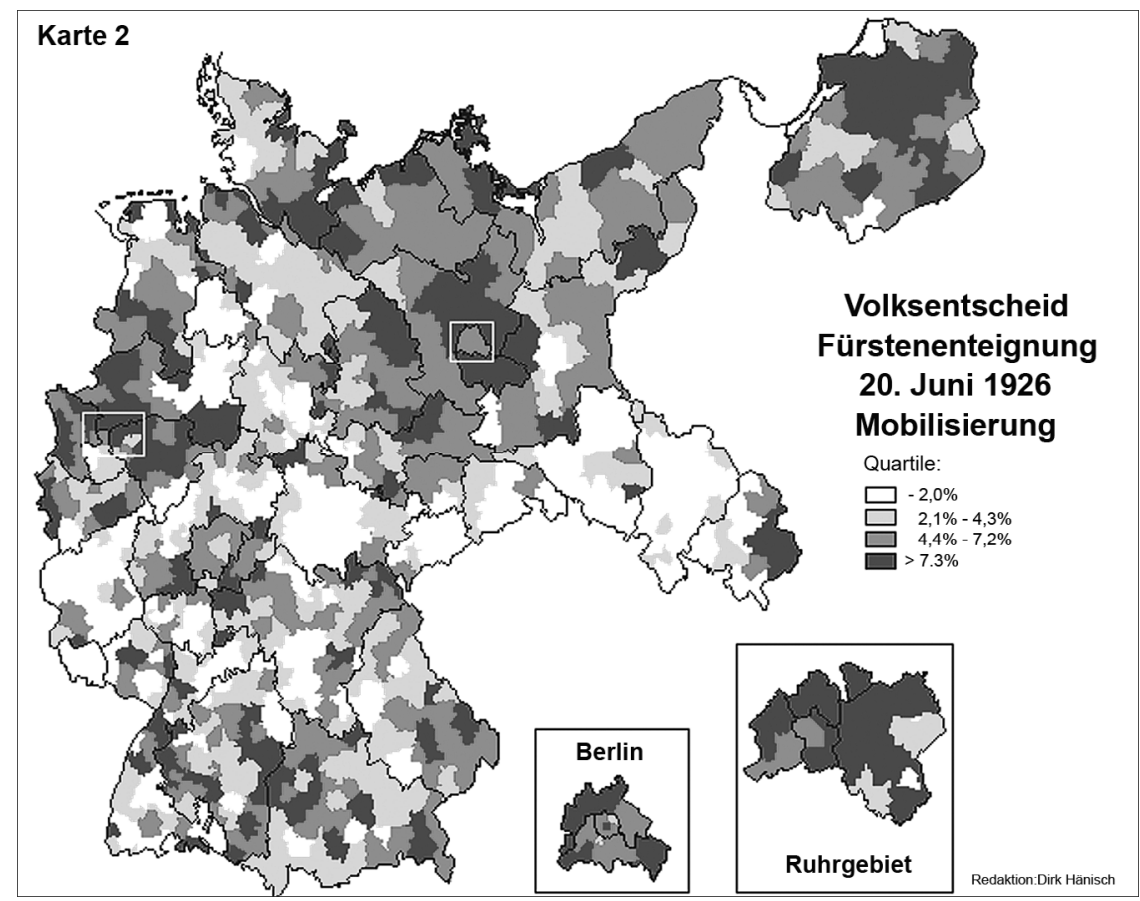

21 Die sozialdemokratische Dresdner Volkszeitung (Dresden) vom 22. Juni 1926 begründete dies wie folgt: „Der Rückgang, der in einem Teil von Sachsen eingetreten ist, ist sicher zum großen Teil zurückzuführen auf die Agitation des Landbundes und der übrigen Fürstenknechte, die mit allen Mitteln versuchten, den kleinen Bauern und Handwerkern vor den Folgen der Fürstenenteignung grausig zu machen. Suchte man doch den Bauern einzureden, daß, wenn die Fürsten enteignet werden, dann auch recht bald dem Bauer die letzte Kuh und das letzte Karnickel durch einen Volksentscheid aus dem Stall geholt werden würde. [...] Schon bei der Agitation für das Volksbegehren hatten die sozialistischen Parteien alles aufgeboten, um auch den letzten Proletarier dazu zu bringen, daß er sich einträgt. Wesentliche Reserven waren in Sachsen im Gegensatz zu andern Landesteilen nicht mehr vorhanden.“ 
b) Die politische Herkunft der Befürworter des Plebiszits von 1926

Aus welchen Lagern und Parteien die Befürworter kamen, war spätestens nach der Ergebnisbekanntgabe des Volksentscheids Gegenstand von Spekulationen, so etwa in den Berichten der Tageszeitungen. Immer wieder wurden quantitative Bezüge zur Reichstagswahl im Dezember 1924 bzw. zu den beiden Wahlgängen der Reichspräsidentenwahl im März/April 1925 hergestellt und Saldierungen und Vergleiche der globalen und lokalen Ergebnisse vorgenommen. Die Zeitgenossen äußerten mit Blick auf die Ergebnisse der zurückliegenden Reichstagswahl vom Dezember 1924 die Vermutung, dass über die beiden Befürworterparteien SPD und KPD hinaus auch aus dem Spektrum der bürgerlichen Parteien für den Volksentscheid votiert wurde, darunter aus Teilen der Zentrumspartei. Insbesondere bestand Konsens darüber, dass zusätzliche Stimmen aus dem eher linksliberalen Wählerspektrum der DDP Stimmen für den Volksentscheid abgegeben wurden, die bekanntlich keine einheitliche Haltung gegenüber dem Plebiszit hatte.

Um die über das linke Lager hinausgehende Wählermobilisierung auch statistisch zum Ausdruck zu bringen, bildete West in seiner Studie über den Volksentscheid Mitte der 1980er Jahre einen Index, der den Grad der Wählermobilisierung über die Initiatorenparteien auf der Linken hinaus misst ${ }^{22}$. Er drückt das Verhältnis der absoluten Ja-Stimmenanzahl beim Volksentscheid zur Summe der SPD-, USPD- und KPD-Stimmen der vorangegangenen Reichstagswahl im Dezember 1924. West verglich die Ergebnisse seiner Indexbildung nach Wahlkreisen sowie einzelnen Stadt- und Landkreisen. Er kam zu dem Ergebnis, dass auch aus der Zentrumswählerschaft Zustimmung für das Plebiszit erfolgte - allerdings ohne eine ungefähre Größenordnung zu nennen. Eine Nachberechnung auf Basis der hier vorliegenden Daten bestätigt diese Aussage. Eigene Berechnungen ergeben darüber hinaus: Der

22 West, Crisis (Fn. 14). Kritisch ist dabei die Tatsache zu sehen, dass er insgeheim damit eine hundertprozentige „Haltequote“ der SPD- und KPD-Wähler unterstellt, was in dieser Höhe sehr unwahrscheinlich ist, denn in den zurückliegenden eineinhalb Jahren dürften auch auf der Linken $\mathrm{Zu}$ - und Abwanderungen stattgefunden haben. Schon 1926 spekulierten auf Basis ähnlicher Berechnungen die Zeitungen. Sehr vereinfachend ging beispielsweise die sozialdemokratische Volksstimme (Hagen) in ihrer Ausgabe vom 24. Juni 1926 vor. Sie errechnete für die katholisch geprägten Wahlkreise den Überschuss gegenüber SPD- und KPD-Stimmen 1924 (Dezember) und ging dann davon aus, dass dieser ausschließlich von Zentrumswählern herrührte: „Wir stellen also fest: Von 3346763 Zentrumswählern haben in den ausgesprochenen Zentrumswahlkreisen nicht weniger als 1267255 bei dem Volksentscheid mit Ja gestimmt. Das sind 38 Prozent der Zentrumswähler in diesen Wahlkreisen.“ 
reichsweite Durchschnitt beträgt $1,418^{23}$ (zum Vergleich: Volksbegehren: 1,1452). Legt man das obere Zehntel der Stimmenanteile (gemessen an den Wahlberechtigten) von Zentrum/BVP als Hochburgen fest, so steigt der Index für diese 127 Gebietseinheiten auf 2,375 (1,5965). Die Schlussfolgerung liegt nahe, dass in diesen überwiegend katholischen Zentrumshochburgen insbesondere beim Volksentscheid überdurchschnittlich gut mobilisiert wurde. Jedoch dürften auch Ja-Stimmen aus dem Nichtwählerlager gekommen sein, denn in deren 127 Hochburgen beträgt der Index immerhin 1,8968 $(1,5487)$. Bestätigt wurde ferner anhand des vorliegenden Datenmaterials, dass die Initiatoren und Unterstützer des Volksentscheids in Pommern aus den genannten Gründen schlechter abschnitten und dort bei der Wählermobilisierung deutlich unter ihren Möglichkeiten blieben.

Lineare Zusammenhangsmaße wie Korrelationen ${ }^{24}$ oder Hochburgenana$\operatorname{lysen}^{25}$ können jedoch keine Hinweise darauf liefern, wie hoch die Anteile der Parteigänger vom Dezember 1924 sind, die Volksbegehren und Volksentscheid befürwortet haben. Dazu eignen sich die Schätzergebnisse nach

23 West kommt auf einen anderen reichsweiten Durchschnittswert $(1,352)$, der rechnerisch so nicht präzise nachvollziehbar ist.

24 Eine bivariate Korrelationsanalyse mit den Stimmenanteilen der Parteien bei der Reichstagswahl im Dezember 1924 ergibt erwartungsgemäß starke positive Beziehungen, die zwar hoch ausfallen, aber keineswegs perfekt sind: SPD: +.631; +.609; -.103 und KPD: $+.595 ;+.630 ;+.098$ (in der Reihenfolge Volksbegehren, Volksentscheid und Mobilisierung). Auch zur DDP bestehen positive lineare Zusammenhänge $(+.438 ;+.443 ;-.002)$. Die übrigen Parteien korrelieren nicht oder eher schwach negativ. Keine ausgeprägten linearen Zusammenhänge gibt es zwischen Parteianteilen und Mobilisierungsquote. Korreliert wurde beim Volksbegehren stets der prozentuale Anteil der Eintragungen an den Stimmberechtigten, beim Volksentscheid der Anteil der Ja-Stimmen an den Stimmberechtigten und bei der Mobilisierung die Differenz der beiden.

25 Definiert man für jede Partei bei der Reichstagswahl im Dezember 1924 das obere Zehntel ihrer Wähleranteile als Hochburgenkreise und errechnet den dort erzielten durchschnittlichen Anteil der Ja-Stimmen beim Volksentscheid, dann werden die folgende Zusammenhänge deutlich: In den 127 SPD-Hochburgen lag die durchschnittliche Unterstützung für den Volksentscheid bei 48,5\% (beim Volksbegehren $45,1 \%$ ), in den KPD-Hochburgen, von denen übrigens nur zwei auch gleichzeitig SPD-Hochburgen waren, immerhin schon 51,2 $(45,9) \%$, bei der DDP 44,4 $(38,9)$ und der DVP 39,4 (34,7)\%, was noch leicht über dem Reichsdurchschnitt von 36,4 $(31,5) \%$ liegt. Anders verhielt es sich bei den Hochburgen der Zentrumspartei/BVP, wo die Zustimmungsquoten bei 19,8 (14,7) bzw. 24,4 (16,6)\% (Zentrum ohne BVP), der DNVP mit 17,5 (12,9)\% und die Nationalsozialistische Freiheitsbewegung mit $28,4(21,8) \%$, die alle deutlich unterhalb des Reichsdurchschnitts von 36,4 (31,5) \% lagen. Auch die Nichtwählerhochburgen rangierten mit 30,1 (25,0)\% Ja-Stimmenanteilen darunter. 
dem Goodman-Verfahren. Sie ergeben beim Volksbegehren für KPD und SPD ähnlich hohe Anteile der Befürwortung von jeweils etwa vier Fünftel ihrer Wähler vom Dezember 1924. Bei der DDP trug sich fast die Hälfte ihrer Wählerschaft in die Listen beim Volksbegehren ein. Beachtlich ist die Aktivierung der vormaligen Nichtwähler. Den Berechnungen zufolge schrieb sich ein knappes Fünftel in die ausgelegten Listen ein. Der offiziellen Parteilinie folgend hielten sich die meisten der über 5,2 Millionen Zentrums- und BVP-Wähler von der Abstimmungsurne fern, denn schätzungsweise nur jeder siebte von ihnen trug sich in die Listen ein.

Beim Volksentscheid steigerte sich vor allem der Anteil der Befürworter aus den Reihen von KPD, DDP und Nichtwählern überdurchschnittlich stark. Die Wählerschaft der DDP bot wieder ein gespaltenes Bild. Auf den ersten Blick scheint der Beitrag der Zentrumspartei mit jedem fünften Wähler reichsweit recht niedrig auszufallen. Stellt man jedoch in Rechnung, dass diese Partei fast ausschließlich von katholischen Wahlberechtigten gewählt wurde, so fiel der Zuspruch zum Volksentscheid im katholischen Segment anteilig sehr viel höher aus als im Reichsdurchschnitt ${ }^{26}$. Es dürften unseren Schätzungen nach umgerechnet knapp eine Million Zentrums-/BVP-Wähler gewesen sein, die für den Volksentscheid votierten, was etwa ein Viertel bis ein Drittel der katholischen Wahlberechtigten ausgemacht haben dürfte. Überraschend ist auch der beachtliche Anteil der mobilisierten ehemaligen Nichtwähler unter den Befürwortern. Die Schätzungen zeigen, dass sowohl die Zeitgenossen als auch die wenigen Historiker, die sich eingehender mit dem Volksentscheid 1926 auseinandersetzten, diese Herkunftsquelle nicht oder kaum im Blick hatten ${ }^{27}$.

26 Das erkennt auch Horstmann, der exemplarisch anhand der Betrachtung dreier katholischer Kreisergebnisse konstatiert, dass die Parteibindung des katholischen Wahlbürgers in dieser Frage lockerer war als die des Stimmbürgers allgemein: „Der ,bekenntnistreue' katholische Enteignungsbefürworter bewies einen wachen Sinn für den situativen Kontext, den eine Politik aus christlicher Verantwortung zu berücksichtigen hat. Wenn die Enteignung weiter Teile des Mittelstandes durch die Inflation rechtens war und wie die Regierung unmißverständlich klar machte, irreversibel, dann war es nur billig, daß auch die depossedierten Fürsten die Folgen des verlorenen Krieges mittrugen." (J. Horstmann, Katholiken, Reichspräsidentenwahlen und Volksentscheide, in: Jahrbuch für Christliche Sozialwissenschaften 27 [1986], S. 61-93 [80]).

27 Schon zahlreiche zeitgenössische Berichterstatter ignorierten in ihrer Wahlnachbetrachtung meistens die Nichtwähler. Das schlägt sich auch oftmals bei der Dokumentation der Wahlergebnisse nieder, wo die Wahlberechtigtenziffer eher als technische und vernachlässigbare Kategorie begriffen wurde. 
Tabelle 1: Schätzungen des Abstimmungsverhaltens bei Volksbegehren und Volksentscheid 1926 (Ja-Stimmenanteil) sowie die parteipolitische Herkunft der Befürworter in gerundeten Prozenten

\begin{tabular}{|l|rr|rr|r|}
\hline 1924, Dezember & \multicolumn{2}{|c|}{$\begin{array}{c}\text { Zustimmung 1926 } \\
\text { beim }\end{array}$} & \multicolumn{2}{c|}{$\begin{array}{c}\text { Parteipolitische } \\
\text { Herkunft der Be- } \\
\text { fürworter 1926 }\end{array}$} & $\begin{array}{c}\text { Reichsweites } \\
\text { RTW-Ergebnis } \\
\text { Dezember 1924 }\end{array}$ \\
\hline KPD & 79 & 93 & 17 & 18 & 7 \\
SPD & 79 & 86 & 51 & 48 & 20 \\
DDP & 46 & 55 & 7 & 7 & 5 \\
DVP & 10 & 10 & 3 & 2 & 8 \\
Zentrum/BVP & 15 & 19 & 6 & 7 & 13 \\
DNVP/NSFB & 0 & 0 & 0 & 0 & 18 \\
Sonstige & 18 & 17 & 3 & 3 & 6 \\
Nichtwähler & 18 & 24 & 13 & 15 & 22 \\
\hline \multicolumn{7}{|r|}{} & & 100 & 100 & 100 \\
\hline
\end{tabular}

Lesebeispiel: 79 Prozent der KPD-Wähler vom Dezember 1924 befürworteten im März 1926 das Volksbegehren und 93 Prozent der KPD-Wähler vom Dezember 1924 stimmten mit Ja beim Volksentscheid (linke Tabellenhälfte). Beim Volksbegehren 1926 stellten die ehemaligen KPD-Wähler 17 Prozent, die SPD-Wähler 51 Prozent usw. der Befürworter. Abweichungen zu 100 sind rundungsbedingt. Abk.: VB = Volksbegehren, VE = Volksentscheid, RTW = Reichstagswahl.

Volksbegehren und Volksentscheid zur Fürstenenteignung waren diesen Ergebnissen nach eine über die Wählerschaft von KPD und SPD hinausreichende Bewegung. Allerdings hielt sich die Einbeziehung von Wählerschichten bürgerlicher Parteien mit Ausnahme der DDP in Grenzen. Das zeigt der Blick auf die parteipolitische Herkunft der Befürworter des Volksentscheids: KPD und SPD stellten gut zwei Drittel der über 14,4 Millionen Befürworter des Volksentscheids, ehemalige Nichtwähler mehr als ein Siebtel und ein knappes Fünftel kam aus dem Spektrum der übrigen Parteien.

c) Das konfessionell-sozioökonomische Profil der Befürworter des Plebiszits 1926

Entsprechend der Erkenntnis, dass die klassischen Cleavagestrukturen einen prägenden Einfluss auf das Wahlverhalten in der Weimarer Republik besaßen, wurden für die Auswertung der Volksabstimmungsergebnisse 1926 und 
1929 aus dem Datenmaterial Kontrastgruppen nach Konfession, Urbanisierung und Wirtschaftsabteilungstyp gebildet. In einem ersten Schritt erfolgte eine Aufgliederung der Städte und Kreise nach dem Konfessionsanteil in einen katholischen, gemischten und protestantischen Cluster. Auf der zweiten Stufe wurde eine Differenzierung nach Stadt/Land eingeführt ${ }^{28}$. Schließlich erfolgte in einem dritten Schritt die Differenzierung der sechs Subgruppen nach der Dominanz des Wirtschaftsabteilungstyps, d.h. Landregionen wurden nach landwirtschaftlicher bzw. nichtlandwirtschaftlicher Mehrheit unterteilt, die Städte hingegen danach, ob es dort eine Mehrheit der industriellhandwerklichen oder des Dienstleistungssektors gab ${ }^{29}$. Somit wurden insgesamt zwölf konfessionell-sozioökonomische Kontrastgruppen generiert, die wichtige Cleavagestrukturen des Weimarer Parteiensystems widerspiegeln. Die zwölf relativ homogenen Kontrastgruppen geben wichtige Hinweise auf das Sozialprofil der Anhänger des Plebiszits. Sie umfassen 1926 insgesamt über 39,7 Millionen Wahlberechtigte, 1929 sind es fast 42,3 Millionen. Grafik 1 stellt die Gliederung der Wahlberechtigten nach den zwölf konfessionell-sozioökonomischen Kontexten für 1926 vor - für 1929 sind die Größenverhältnisse nur geringfügig anders. Die größte Gruppe der Wahlberechtigten im Deutschen Reich stellte demnach das protestantische Land mit nichtlandwirtschaftlicher Mehrheit, dann die evangelischen Städte mit nichtindustrieller Dominanz. Mit größerem Abstand folgten die protestantischen Gegenstücke: die bäuerlichen Landgebiete und die industrie- und handwerksdominierten Städte. Demgegenüber nahmen die katholischen Stadt- und Landregionen eine relativ marginale Position ein.

28 Als protestantisch bzw. katholisch wurden hier alle Städte und Gemeinden mit einem Protestanten- bzw. Katholikenanteil von mehr als zwei Dritteln der Wohnbevölkerung bezeichnet. Die übrigen Städte und Kreise zählen zu den gemischten Konfessionstypen. Als urbane Regionen wurden neben Städten solche Kreise identifiziert, deren Anteil an Gemeinden mit 5.000 oder mehr Einwohnern bei mindestens 75 Prozent lag. Diese Klassifizierung hat sich bereits bei früheren Analysen des Wahlverhaltens in der Weimarer Republik als recht trennscharf erwiesen, vgl. dazu die Beispiele bei Falter u.a., Wahlen (Fn. 1), S. 200 und S. 203 (dort Fn. 1).

29 Die Volks- und Berufszählung 1925 unterteilt die Erwerbstätigen und ihre Angehörigen in die Wirtschaftsabteilungen Land- und Forstwirtschaft, Fischerei; Industrie und Handwerk; Handel und Verkehr; Verwaltung, Heerwesen, Kirche, freie Berufe; Gesundheitswesen, hygienische Gewerbe, Wohlfahrtspflege; Häusliche Dienste, Erwerbstätigkeit ohne feste Stellung oder ohne Angabe der Betriebszugehörigkeit. Zum Dienstleistungssektor wurden hier alle nichtlandwirtschaftlichen Wirtschaftsabteilungen außer Industrie und Handwerk gezählt. 


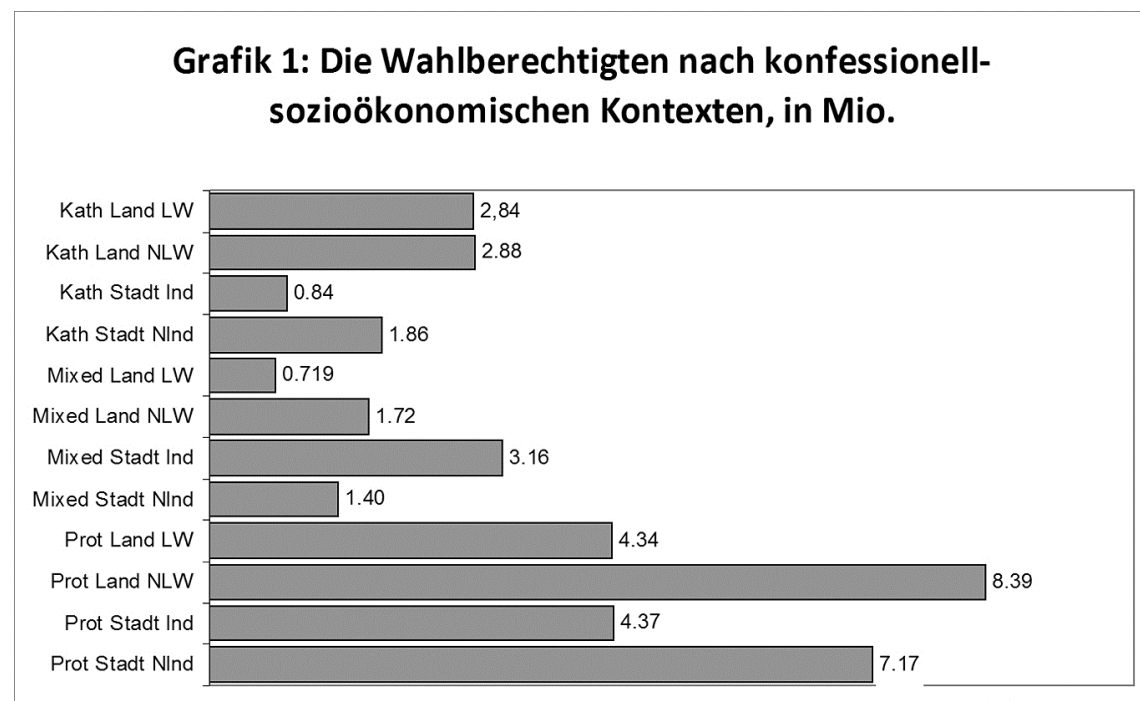

Abk.: Kath $=$ Katholische Mehrheit; Mixed $=$ Gemischt konfessionell; Prot $=$ Protestantische Mehrheit; LW = Landwirtschaftliche Dominanz; NLW = Nichtlandwirtschaftliche Dominanz; Ind = Dominanz Industrie/Handwerk; NInd = Dominanz Dienstleistungen.

In Grafik 2 sind die durchschnittlichen Prozentanteile bei Volksbegehren und Volksentscheid sowie der Mobilisierungsgrad nach konfessionell-sozioökonomischen Kontexten grafisch abgetragen. Ergänzend dazu wurde der gemeinsame Wähleranteil der Hauptinitiatoren SPD und KPD bei der vorangegangenen Reichstagswahl 1924 (Dezember) errechnet. Die besten Ergebnisse beim Plebiszit 1926 wurden in Städten mit Industrie- und Handwerksmehrheit erzielt, und zwar in der Reihenfolge protestantisch, gemischt und katholisch. Es zeigt sich außerdem, dass der Zuspruch zum Volksentscheid im Juni 1926 im Kontext „Protestantisches Land mit landwirtschaftlicher Dominanz“ aus den bereits erwähnten Gründen unter dem gemeinsamen Wahlergebnis von KPD und SPD von Ende 1924 lag; hingegen fiel er in allen übrigen Kontexten stets höher aus. In den übrigen protestantischen Kreisen und Städten wurde beim Volksentscheid nur noch mäßig hinzugewonnen, in gemischten und katholischen Kreisen dagegen etwas stärker. Möglicherweise kamen aus diesem Segment unter dem Eindruck des erfolgreich verlaufenen Volksbegehrens vermehrt Stimmen von städtischen Zentrumswählern. 
Grafik 2: Prozentanteile beim Plebiszit 1926 nach konfessionellsozioökonomischen Kontrastgruppen

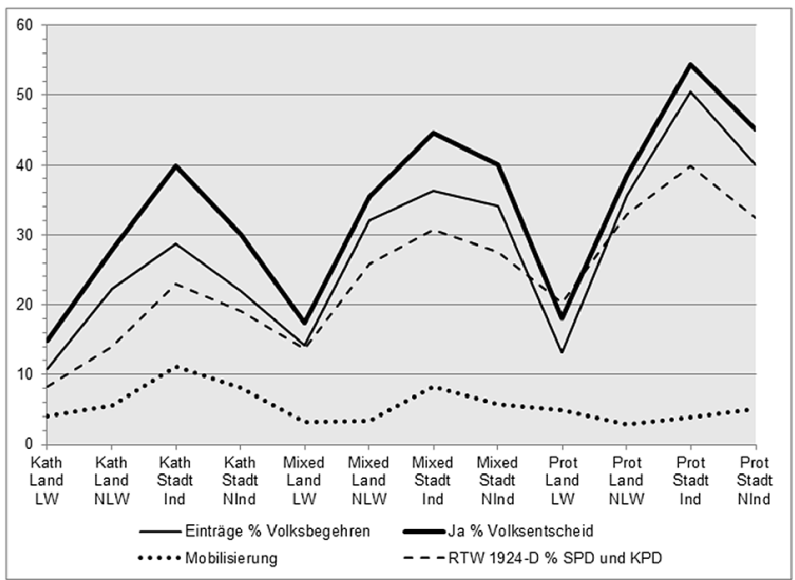

d) Das Sozialprofil der Befürworter des Plebiszits von 1926

Erste Hinweise auf das soziale Profil der Plebiszitbefürworter können mit Hilfe von Korrelationen der Berufszählungsdaten von 1925 für die Kreise und Städte des Deutschen Reiches gewonnen werden. Dieses Vorhaben ist methodisch und statistisch nicht ganz unproblematisch. Zum einen sind die Volkszählungskategorien relativ vage und amorph ${ }^{30}$, zum anderen sind die Bezugsbasen bei den Wahl- und Sozialdaten unterschiedlich (Wahlberechtigte versus Wohnbevölkerung). Ähnliche Einschränkungen und Hilfsannahmen müssen natürlich mit Blick auf Wanderungsbewegungen, $\mathrm{Zu}$ - und Abgänge sowie andere Veränderungen des Wahlkörpers getroffen werden, die hier datenmäßig nicht abgebildet werden können. Diese Ungenauigkeiten und me-

30 „Die Spannweite der Lebensumstände und Arbeitsverhältnisse, die sich hinter dem Sammelbegriff ,Arbeiter' verbargen, war nicht viel geringer als bei den Angestellten und Beamten, die in der Nomenklatur der Volkszählung vom Büropersonal bis zum Filialleiter und vom Amtsboten bis zum Regierungsdirektor eine ganze Skala unterschiedlicher Berufe und Soziallagen umfaßten“, J.W.Falter/D. Hänisch, Die Anfälligkeit von Arbeitern gegenüber der NSDAP bei den Reichstagswahlen 1928-1933, in: Archiv für Sozialgeschichte 26 (1986), S. 179-216 (179). 
thodischen Mängel sind unvermeidlich, wenn überhaupt annähernde Aussagen über das Wahlverhalten getroffen werden sollen. Auch aus diesem Grund sollten die hier präsentierten Schätzergebnisse nur holzschnittartig als annähernde Größenordnungsverhältnisse angesehen werden.

Die Korrelationen mit den wichtigsten Berufszählungsanteilen, die die soziale Stellung ${ }^{31}$ nach Wirtschaftsabteilungszugehörigkeit beschreiben, verdeutlichen zum einen die starke Polarisierung entlang der Wirtschaftsabteilungen Landwirtschaft versus Industrie/Handwerk, zum anderen eine ebenfalls starke Polarisierung zwischen Selbständigenanteilen auf der einen und den Angestellten- bzw. Arbeiteranteilen auf der anderen Seite ${ }^{32}$. Untergliedert man die Wirtschaftsabteilungen nach sozialer Stellung, dann verläuft die Spannungslinie bei diesem Plebiszit zwischen Bauern und Landarbeitern einerseits und den nichtlandwirtschaftlichen Angestellten und Arbeitern andererseits.

Eine weitere Annäherung zur Beantwortung der Frage nach dem Sozialprofil der Befürworter des Plebiszits kann durch den vergleichenden Blick auf Hochburgen und Nichthochburgen der Plebiszitbefürworter erfolgen. Wenn als Hochburgenkriterium das obere Zehntel vereinbart wird, dann kann das sozialstrukturell-konfessionelle Profil dieser 127 Städte, Kreise und Verwaltungsbezirke mit den übrigen Kreisen und Städten verglichen werden. An der Spitze stehen Groß Ottersleben (Wahlkreis 10 mit 78,3 Prozent bei 61,1 Prozent SPD/KPD-Anteil), Neugersdorf (Wahlkreis 28 mit 75,8 Prozent versus 47,5 Prozent), Berlin-Wedding (Wahlkreis 2 mit 74,3 Prozent versus 49,8 Prozent), Höhscheid (Wahlkreis 22 mit 72,7 Prozent versus 44,1 Prozent) und Berlin-Neukölln (Wahlkreis 3 mit 72,2 Prozent versus 48,2 Prozent). Ein solcher Vergleich der Ja-Stimmenanteile beim Volksentscheid zeigt dann teilweise erhebliche Unterschiede. In konfessioneller Hinsicht waren die Hochburgen des Volksentscheids mit 14,6 Prozent deutlich weniger katholisch als die Nichthochburgen (34,3 Prozent). Die oben skizzierte Kon-

31 Die soziale Gliederung wird in der Volks- und Berufszählung nach der Stellung im Beruf aufgeschlüsselt, und zwar in Selbständige (a-Personen), Angestellte und Beamte (b), Arbeiter (c) und Mithelfende (m), jeweils nach Wirtschaftsabteilungen. Hinzu kommen die Personen ohne Beruf und Berufsangabe, also Berufslose. Von diesen lebten mehr als drei Viertel von Renten und Pensionen, hinzu kamen Studierende und Schüler. Zur Systematik der Berufszählung 1925 vgl. Statistik (Fn. 2), Bd. 402, II (Berlin 1927), S. 215 ff.

32 Gewichtete Korrelationen (nach Pearson) zwischen Ja-Stimmenanteil und Landwirtschaftsanteil -.732 bzw. mit Industrie-/Handwerksanteil +.703 ; mit dem landwirtschaftlichen Selbständigenanteil -.698, dem Angestellten-/Beamtenanteil +.511 und dem nichtlandwirtschaftlichen Arbeiteranteil +.750. 
fliktlinie zwischen Landwirtschaftssektor und Industrie/Handwerk schlägt sich wie folgt nieder: In den 127 Hochburgen waren im Schnitt nur drei Prozent der Bevölkerung dem Landwirtschaftssektor zugehörig (versus 25,2 Prozent), 55,7 Prozent zählten in den Hochburgen zum Industrie- und Handwerkssektor (versus 39,7 Prozent), und 33,1 Prozent (versus 25,9 Prozent) waren zum Dienstleistungssektor zu zählen. Die Befürworter des Volksentscheids kamen demzufolge aus den wirtschaftlich entwickelteren Sektoren, die Gegner bzw. Indifferenten repräsentierten tendenziell eher das wirtschaftlich rückständigere und weniger modernisierte Deutschland. Der Gegensatz von Kapital und Arbeit, der in dieser ersten Hälfte des 20. Jahrhundert eine beherrschende Rolle spielte, spiegelte sich in der Fürstenenteignungsfrage und fand im Plebiszit wie unter einem Brennglas seinen symbolischen und ideologisch hoch aufgeladenen Ausdruck. Das zeigt sich schließlich auch bei einem Vergleich hinsichtlich der sozialen Stellung. In den Hochburgen war der nichtlandwirtschaftliche Arbeiteranteil mit 49,5 Prozent sehr hoch (in den Nichthochburgen betrug er 34,7 Prozent), bei den Angestellten und Beamten war das Verhältnis 22,3 Prozent zu 15,7 Prozent. Entsprechend lagen die Anteile des landwirtschaftlichen Personals in den Hochburgen des Volksentscheids deutlich niedriger: landwirtschaftliche Selbständige 1,9 Prozent (versus 17,7 Prozent) und Landarbeiter 1,0 Prozent (versus 7,0 Prozent).

Ein Kontrastgruppenvergleich ${ }^{33}$ aller Städte und Kreise nach ihrer Konfessionszugehörigkeit und nichtlandwirtschaftlichen Arbeiteranteilen ${ }^{34}$ verdeutlicht, wie stark das Ergebnis der Volksabstimmung vom Anteil der nichtlandwirtschaftlichen Arbeiter in allen konfessionellen Kontexten mitbestimmt wurde, auch wenn der Zuspruch in den katholischen Städten und Kreisen größenmäßig stets niedriger liegt (Grafik 3 auf der nächsten Seite). In der Subgruppe der protestantischen Kreise mit sehr hohen nichtlandwirtschaftlichen Arbeiteranteilen wurde im Schnitt sogar eine Mehrheit beim Volksentscheid (50 bzw. fast 53 Prozent) erzielt, während die Zustimmung in der katholischen Vergleichsgruppe deutlich darunter lag (knapp 28 bzw.

33 Das Verfahren des Kontrastgruppenvergleichs wird anschaulich erläutert bei Falter u.a., Wahlen (Fn. 1), S. 190 ff.

34 Gemeint sind hier mit ,nichtlandwirtschaftlichen Arbeitern“ die gemeinsamen Anteile der Arbeiter der Wirtschaftsabteilungen Industrie und Handwerk sowie Handel und Verkehr. Die übrigen nichtlandwirtschaftlichen Arbeiter, die zusammengenommen mit ihren Angehörigen weniger als zwei Prozent ausmachen, bleiben hier unberücksichtigt. 


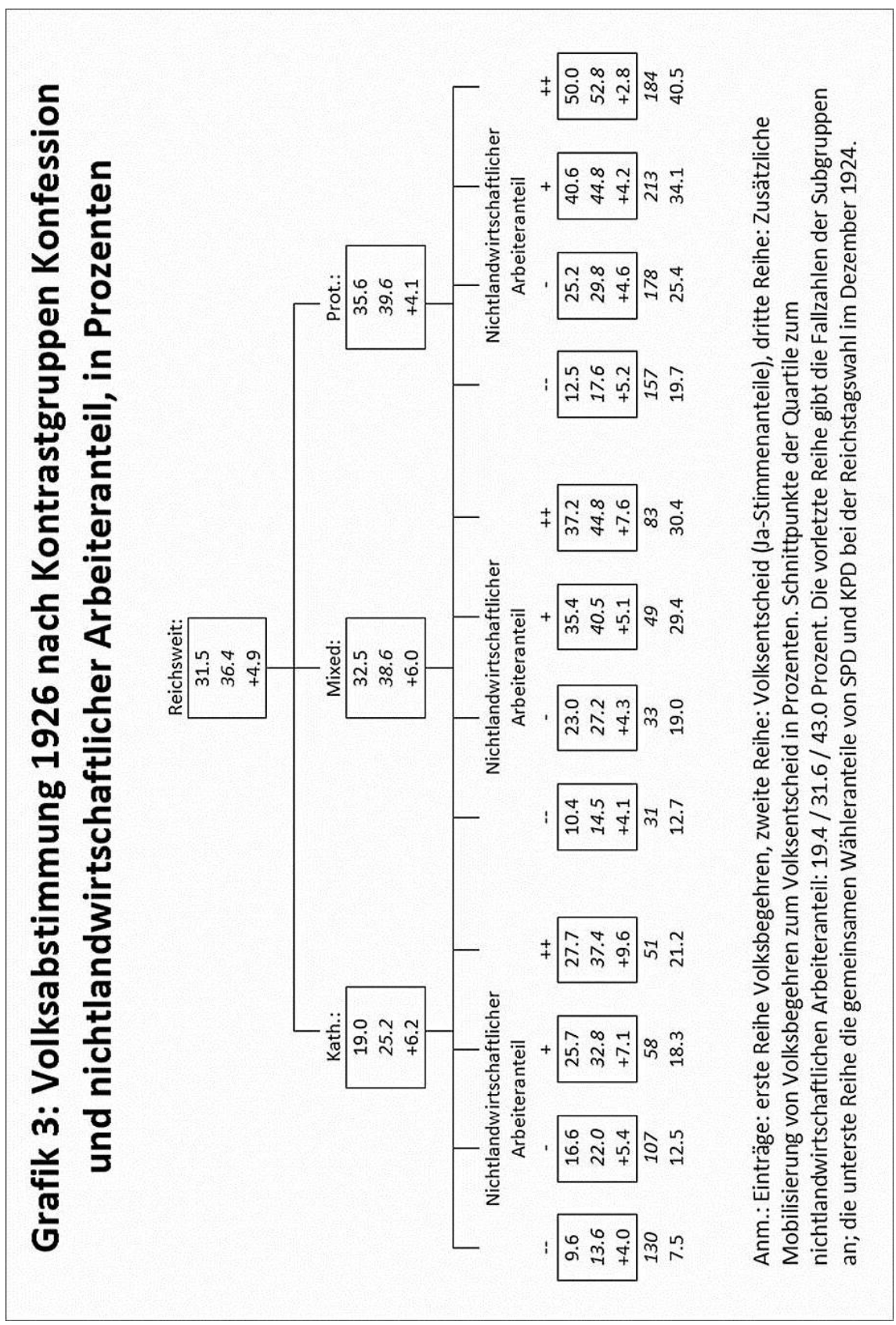

über 37 Prozent). Waren in den protestantischen Kreisen gewissermaßen die 
Mobilisierungsmöglichkeiten ziemlich ausgeschöpft, so konnten in den katholischen und gemischt konfessionellen Kreisen mit einem überdurchschnittlichen nichtlandwirtschaftlichen Arbeiteranteil noch hohe Zuwächse beim Volksentscheid verzeichnet werden. Die strukturellen Unterschiede wurden dadurch aber keineswegs ausgeglichen, sondern blieben grundsätzlich bestehen.

Der überdurchschnittlich hohe Zuspruch der nichtlandwirtschaftlichen Arbeiter wird durch eine ökologische Schätzung im Kern bestätigt. Das Volkszählungsmaterial ermöglicht eine Aufgliederung in drei Gruppen: Arbeiter in Industrie und Handwerk, Arbeiter in Handel und Verkehr sowie Landarbeiter und sonstige Arbeiter. Die beiden Gruppen der nichtlandwirtschaftlichen Arbeiter, aber auch die Angestellten/Beamten votierten dieser Schätzung nach jeweils gut zur Hälfte für die Fürstenenteignung und waren dadurch anteilig überdurchschnittlich hoch am Ja-Stimmenkontingent beteiligt. Dem gegenüber besaßen landwirtschaftliche Arbeiter und noch stärker Selbständige und Mithelfende deutlich größere Ressentiments gegenüber dem Volksentscheid $^{35}$.

35 Darüber schrieb die rechtsgerichtete Allgemeine Zeitung der Lüneberger Heide (Uelzen): „Also hier auf dem Lande die umgekehrte Erscheinung wie bei der Großstadtbevölkerung. Dort begeistern sich auch außer den Marxisten noch allerlei andere Leute [...] für eine beginnende Bolschewisierung, bei der Landbevölkerung ist dagegen die Unverletzlichkeit des Eigentumbegriffs so fest in Denken und Trachten verankert, daß sogar bis in die Kreise derjenigen, die sonst marxistisch zu stimmen pflegen, dieser Gedanke einer entschädigungslosen Enteignung abgelehnt wurde.“ 
Tabelle 2: Geschätztes Abstimmungsverhalten nach sozialer Stellung bei Volksbegehren und Volksentscheid 1926 (Ja-Stimmenanteil) sowie die soziale Herkunft der Befürworter in gerundeten Prozenten

\begin{tabular}{|c|c|c|c|c|c|}
\hline & \multicolumn{2}{|c|}{$\begin{array}{l}\text { Zustimmung } \\
1926\end{array}$} & \multicolumn{2}{|c|}{$\begin{array}{c}\text { Soziale Herkunft } \\
1926\end{array}$} & \multirow{2}{*}{$\begin{array}{c}\text { Berufs- } \\
\text { zählung } \\
1925\end{array}$} \\
\hline & VB & VE & VB & VE & \\
\hline $\begin{array}{l}\text { Selbständige und Mithelfen- } \\
\text { de }\end{array}$ & 14 & 15 & 14 & 12 & 30 \\
\hline Angestellte und Beamte & 49 & 56 & 26 & 26 & 17 \\
\hline $\begin{array}{l}\text { Industrie-/Handwerks- } \\
\text { arbeiter }\end{array}$ & 49 & 57 & 39 & 47 & 30 \\
\hline Handel-/Verkehrsarbeiter & 44 & 52 & 9 & 7 & 5 \\
\hline LW- und sonstige Arbeiter & 29 & 34 & 12 & 8 & 8 \\
\hline \multirow[t]{2}{*}{ Berufslose } & 0 & 0 & 0 & 0 & 11 \\
\hline & & & 100 & 100 & 100 \\
\hline
\end{tabular}

Lesebeispiel: Von den Selbständigen befürworteten im März 192614 Prozent das Volksbegehren und 15 Prozent stimmte beim Volksentscheid mit Ja (linke Tabellenhälfte). Von den Befürwortern beim Volksbegehren 1926 stellten die Selbständigen 14 Prozent, die Angestellten 26 Prozent usw. Abweichungen zu 100 sind rundungsbedingt.

Die Befunde stimmen recht gut mit einer Schätzung des Wahlverhaltens nach sozialer Schichtzugehörigkeit bei der vorangegangenen Reichstagswahl im Dezember 1924 überein, die hier nur gestreift werden kann. Die Industrieund Handwerksarbeiter zählten zur Kernklientel von SPD und KPD. Die auf gleicher Datenbasis, aber nach dem Logit-Schätzverfahren von Thomsen errechneten Wahlpräferenzen der industriellen und handwerklichen Arbeiter fielen demnach am stärksten für die SPD (fast 34 Prozent) und die KPD (13 Prozent) aus. Umgekehrt ausgedrückt: Industrie- und Handwerksarbeiter stellten fast die Hälfte der SPD- bzw. fast drei Fünftel der KPD-Wähler ${ }^{36}$. Von den Angestellten und Beamten stimmten den Schätzziffern zufolge etwa 20 Prozent für die SPD und zehn Prozent für die KPD ${ }^{37}$.

36 Den Schätzungen nach votierten für die Zentrumspartei etwa zwölf Prozent der Industrie- und Handwerksarbeiter, und etwa 16 Prozent blieben der Reichstagswahl im Dezember 1924 fern.

37 Zur Erinnerung: Angestellte und Beamte wurden bei der Volkszählung 1925 zusammen ausgewiesen. 
3. Volksbegehren und Volksentscheid im Oktober bzw. Dezember 1929 gegen den Young-Plan (,Versklavung des deutschen Volkes“)

Ganz anders gestalteten sich die Zusammenhänge beim Volksbegehren und Volksentscheid in der zweiten Jahreshälfte 1929. Die Initiative ging von der politischen Rechten um Hugenberg und seiner DNVP aus. Das Plebiszit wurde vom Stahlhelm um Seldte sowie von den damals noch relativ bedeutungslosen Nationalsozialisten unterstützt ${ }^{38}$. Es richtete sich vordergründig gegen die Höhe und die drakonischen Bedingungen der Reparationszahlungen im Young-Plan. Im Kern lief es auf den Versuch hinaus, den Versailler Vertrag vollkommen zu revidieren.

Das Volksbegehren dazu fand vom 16. bis 29. Oktober 1929 statt und erreichte mit 10,02 Prozent (4,1 Mio.) ganz knapp die geforderte Mindestquote an Zustimmung bei den Stimmberechtigten ${ }^{39}$. Beim Volksentscheid am 22. Dezember 1929 stimmten 5,8 Mio. mit Ja, das waren 13,8 Prozent der Stimmberechtigten - immerhin eine Steigerung um ein Drittel gegenüber Ende Oktober. Dennoch enttäuschte das Ergebnis die Befürworter und Verfechter des Plebiszits, nicht zuletzt, weil die Linke beim mehr als zwei Jahre zurückliegenden Volksbegehren und Volksentscheid mit 14,4 Millionen weit mehr Stimmberechtigte mobilisieren konnte. Mancherorts fiel der Ausgang so niedrig und vernichtend schlecht aus, dass die lokale Presse es vorzog, über den Verlauf des zeitgleich stattfindenden verkaufsoffenen „Goldenen Sonntags“ ausführlicher als über den Ausgang des Volksentscheides zu berichten, der lediglich mit einer kleinen Randnotiz Erwähnung fand.

38 Vgl. dazu bei Falter, Wähler (Fn. 8), S. 36, wo eine Grafik mit dem Anstieg der Nationalsozialisten bei den Wahlen in Reich und Ländern in der Weimarer Republik dargestellt ist. Der „takeoff-point“ (vgl. E. Faris, Takeoff Point for the National Socialist Party: The Landtag Election in Baden, 1929, in: Central European History 8 [1975], S. 140-171) der Nationalsozialisten ist demnach auf etwa Ende Oktober 1929 zu lokalisieren, fast zeitgleich zusammenfallend mit dem Tod Gustav Stresemanns. Seit dieser Landtagswahl in Baden errangen die Nationalsozialisten bei allen Folgewahlen deutlich an Stimmen und hätten dann die - wenn es sie gegeben hätte Fünf-Prozent-Klausel jedes Mal locker übersprungen.

39 Zugrunde lagen dieser offiziellen Berechnung für das Volksbegehren die Wahlberechtigtenzahlen der vorangegangenen Reichstagswahl 1928. Bezogen auf die Stimmberechtigtenziffer des Volksentscheids im Dezember 1929, die zeitlich näher liegt und Basis dieser Analysen ist, waren es 9,8 Prozent an Eintragungen von Stimmberechtigten. 
a) Die regionale Herkunft der Befürworter des Plebiszits 1929

Der Volksentscheid 1929 stieß im protestantischen Osten Deutschlands auf erheblich bessere Resonanz als in den katholischen Gegenden oder den industrialisierten Regionen der Mitte und des Westens. Die Hochburgen, die Karte 3 ausweist, lagen neben Ostpreußen und Pommern auch in Teilen Brandenburgs und im Norden Deutschlands sowie in beiden Mecklenburg. Verglichen mit dem Ausgang des Plebiszits von 1926 verhielt es sich fast spiegelbildlich umgekehrt: Überall dort, wo der Volksentscheid 1926 erfolgreich war, lagen 1929 die Diasporagebiete und umgekehrt. Die Spannweite reichte bei den Städten und Kreisen von 0,17 bzw. 0,18 Prozent Zustimmung in Kohlscheid und Eilenburg (beide Landkreis Aachen) bis hin zu 67,7 bzw. 76,1 Prozent in den Kreisen Uffenheim und Rothenburg ob der Tauber (beide Mittelfranken).

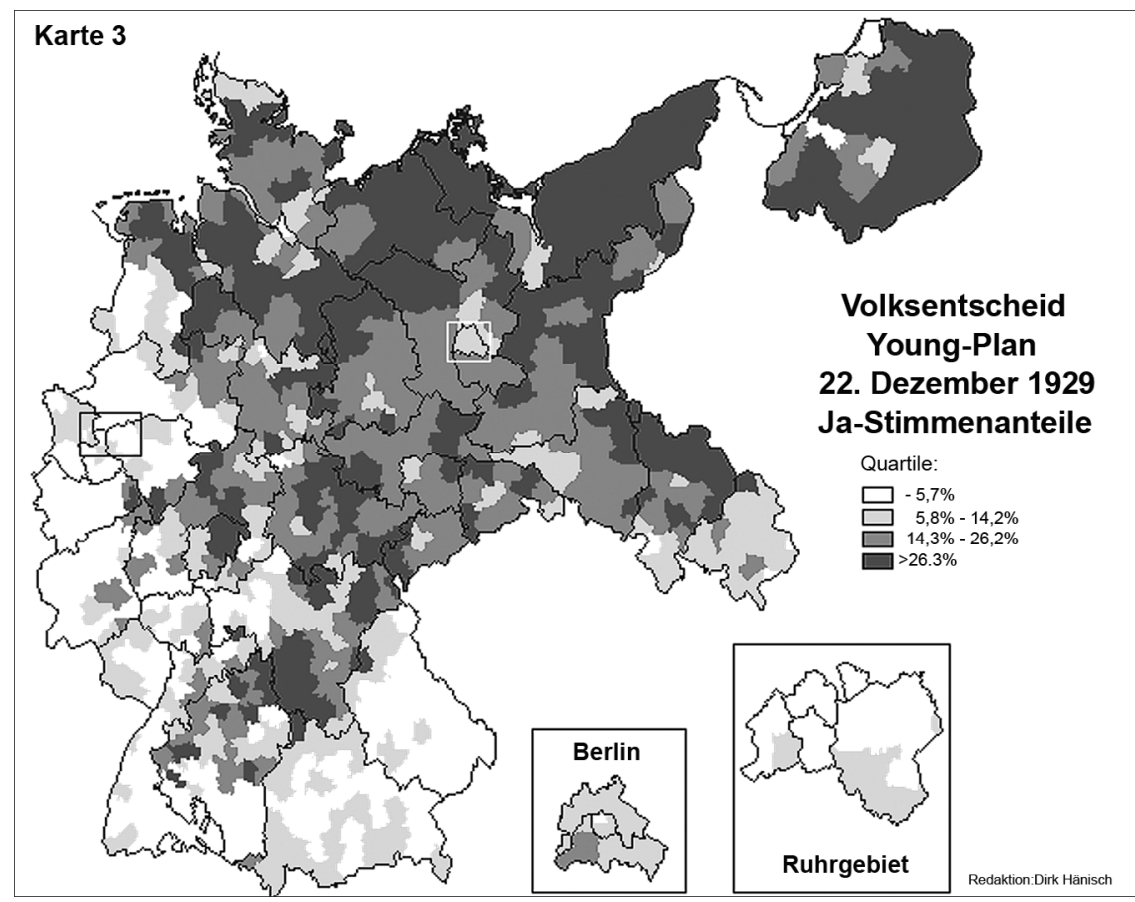


Ein Blick auf die Mobilisierungskarte (Karte 4) zeigt, wo neue Befürworter für den Volksentscheid überdurchschnittlich erfolgreich gewonnen wurden.

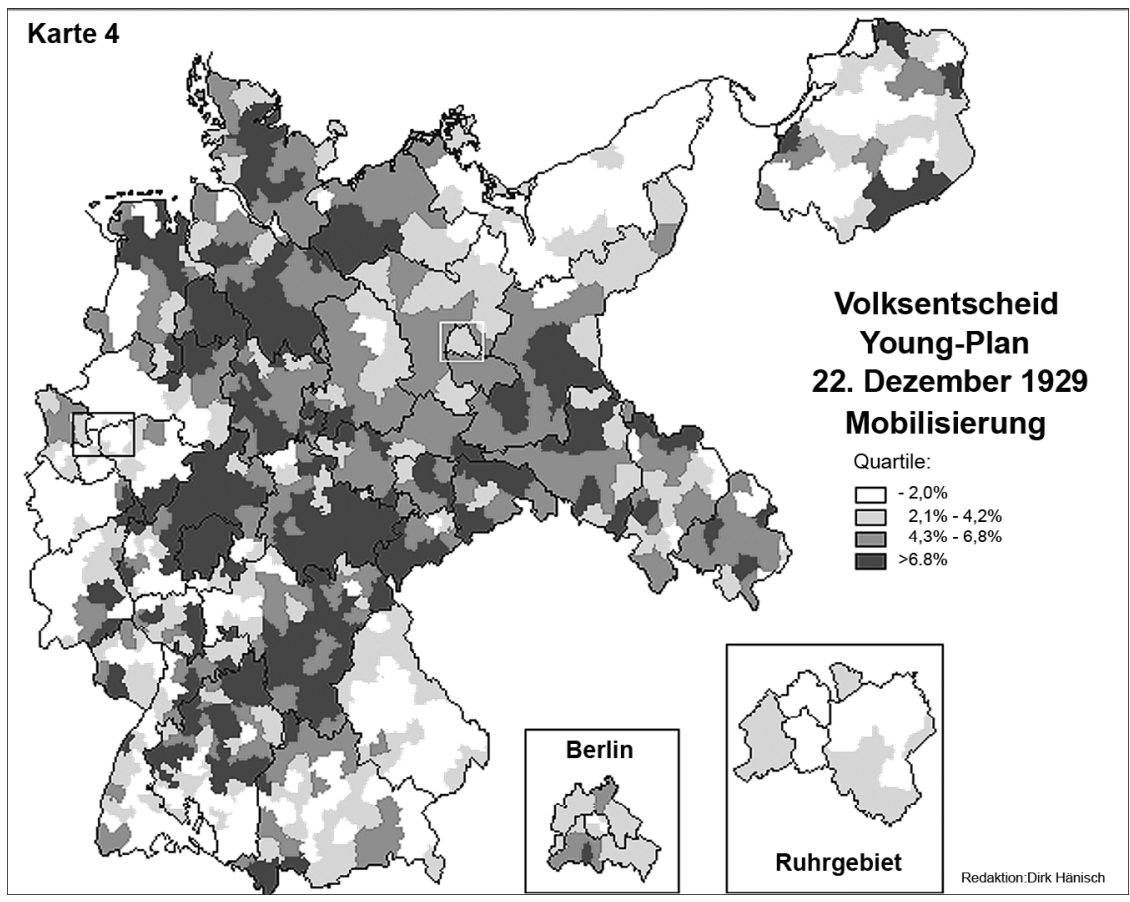

Im Osten Deutschlands, vor allem in Pommern ${ }^{40}$, war mit wenigen Ausnahmen das Mobilisierungspotenzial durch das Volksbegehren weitgehend aus-

40 Die Frankfurter Zeitung berichtete mehrmals über die sozialen und politischen Herrschaftsverhältnisse und die politische Rückständigkeit in Pommern, insbesondere über die gesellschaftliche Vormachtstellung der protestantisch-deutschnationalen Gutsbesitzer, die ihre Untergebenen zum Einzeichnen nötigten. In einem Kommentar auf ihrer ersten Seite am 5. November 1929 (Morgenblatt) unter der Überschrift „Im Reiche des Gutsherrn“ schrieb sie anschaulich über den Zwang zur Einschreibung in die Listen, der in diesen Gegenden ausgeübt wurde: „Auf Leiterwagen wurden die Männer mit ihren Familien stellenweise geschlossen zum Gemeindehaus gefahren, um sie beim Unterschreiben kontrollieren zu können. Die Inspektoren, die Gutssekretäre und sonstige Vertraute mußten jeden einzelnen solange bearbeiten, bis er sich zur Leistung der Unterschrift herbeiließ. Die schwierigsten Fälle behandelte der Amtsvorsteher in höchsteigener Person [...] Hühnermädchen und Chauffeur, Mamsell und Schweinefütterer, Stütze und Kutscher, Gesellschafterin und Gutsschmied, Haustochter und Schnapsbrenner, Gänsemädchen und Förster, oder wie sie sich sonst alle nennen, mußten unbedingt zur Unterschrift heran.“ 
geschöpft. In diesen Hochburgen fielen die Zuwächse beim Volksentscheid meist unterdurchschnittlich aus - ganz ähnlich wie im Fall des Plebiszits 1926 z.B. in Sachsen. Mobilisierungserfolge gab es dagegen vor allem in verschiedenen Teilen der Mitte Deutschlands, so insbesondere im mittleren Schleswig-Holstein, großen Teilen des nichtkatholischen heutigen Niedersachsens, in Thüringen und im Volksstaat Hessen sowie im südlichen Hessen-Nassau, Franken und Teilen von Württemberg. Erkennbar waren es eher die protestantischen Regionen, wo die Mobilisierung erfolgreicher verlief.

\section{b) Die politische Herkunft der Befürworter des Plebiszits von 1929}

Das Korrelationsmuster der Parteianteile bei der Reichstagswahl 1928 mit den Ergebnissen des Volksbegehrens, des Volksentscheides sowie der Mobilisierung 1929 belegen die Verankerung der Befürworter des Plebiszits im Umfeld der DNVP. Ihre Anteile korrelieren stark mit der Zustimmung zum Plebiszit, die NSDAP-Wähleranteile sind beim Volksentscheid moderat bis schwach positiv assoziiert. Alle übrigen Parteien lassen keine linearen $\mathrm{Zu}-$ sammenhänge zum Plebiszitausgang erkennen; Ausnahme sind KPD und Zentrum/BVP mit schwach negativen Korrelationen ${ }^{41}$. Hinsichtlich der Mobilisierung zum Volksentscheid korrelieren die NSDAP-Anteile schwach positiv mit dem Mobilisierungspotenzial.

Auch hier belegt eine Hochburgenanalyse, dass das Plebiszit 1929 fast ausschließlich von DNVP und NSDAP getragen wurde. In den 111 Städten und Kreisen, die das obere Zehntel der Stimmenanteile der DNVP bei der Reichstagswahl im Mai 1928 bildeten, betrugen die Ja-Stimmen beim Volksentscheid 38,7 Prozent, bei der NSDAP 22,7 Prozent und bei der Reichspartei des Deutschen Mittelstandes (Wirtschaftspartei) 19,1 Prozent - alle fallen überdurchschnittlich aus bei einem Reichsdurchschnitt von 13,8 Prozent $^{42}$. Die höchsten Zuwächse beim Volksentscheid erfolgten dabei in den Hochburgen der NSDAP mit 6,7 und der Wirtschaftspartei 6,2 Prozent - Mobili-

41 In Korrelationskoeffizienten ausgedrückt heißt das: DNVP +.761; +.693; +.026; NSDAP +.188; +.253; +.298; KPD -.212; -.252; -.235 und Zentrum/BVP - .435; .468 ; -.272 (erste Ziffer: Volksbegehren, zweite Ziffer: Volksentscheid, dritte Ziffer: Mobilisierungsquote).

42 Zur Ergänzung: In den Hochburgen von SPD 14,2 Prozent, DVP 13,8, DDP 11,3, KPD 9,5 und Zentrum/BVP 3,9 Prozent. Bei den Nichtwählern waren es 11,8 Prozent. 
sierungswerte, die doppelt so hoch liegen wie die in den DNVP-,,strongholds" (3,1 Prozent).

Eine Schätzung der Wählerbewegungen von der Reichstagswahl 1928 zum Plebiszit 1929 ergibt, dass nur etwa zwei Drittel der DNVP-Wähler von 1928 beim Volksentscheid 1929 mit Ja gestimmt hat. Ein weiterer nennenswerter Zufluss erfolgte aus den Reihen der nationalsozialistischen Wähler von 1928, von denen etwa zwei Fünftel mit Ja stimmte. Ferner votierten zu je einem Drittel die Wähler der Reichspartei des Deutschen Mittelstands (Wirtschaftspartei) ${ }^{43}$ und des Landvolks; ferner ein Fünftel der Wähler der sonstigen Parteien, darunter die Volksrechtspartei, für das Volksbegehren. Das gesamte rechte Spektrum jenseits der DVP fühlte sich vom Volksentscheid angesprochen, wenn auch in deutlich geringerem Ausmaß als erwartet. Anders als beim linken Volksentscheid im Juni 1926 blieben bei diesem Plebiszit die Nichtwähler zuhause. Und im Gegensatz zur Reichstagswahl 1930, wo die NSDAP von den Wählerabflüssen der beiden liberalen Parteien profitierten konnte, blieb deren Wählerschaft Ende 1929 weitgehend resistent gegenüber dem deutschnationalen Volksbegehren und Volksentscheid.

Tabelle 3: Geschätztes Abstimmungsverhalten bei Volksbegehren und Volksentscheid 1929 (Ja-Stimmenanteil) sowie die parteipolitische Herkunft der Befürworter in gerundeten Prozenten

\begin{tabular}{|l|rr|rr|r|}
\hline 1928, Mai & \multicolumn{2}{|c|}{$\begin{array}{c}\text { Zustimmung 1929 } \\
\text { beim }\end{array}$} & \multicolumn{2}{|c|}{$\begin{array}{c}\text { Parteipolitische Herkunft } \\
\text { der Befürworter 1929 }\end{array}$} & $\begin{array}{c}\text { RTW-Ergebnis } \\
\text { Mai 1928 }\end{array}$ \\
\hline VB & VE & VB & VE & 8 \\
DDP & 0 & 0 & 0 & 0 & 4 \\
DVP & 5 & 8 & 2 & 2 & 7 \\
Zentrum/BVP & 10 & 16 & 6 & 8 & 11 \\
RdM & 3 & 4 & 3 & 4 & 3 \\
DNVP & 22 & 35 & 8 & 8 & 11 \\
NSDAP & 52 & 65 & 57 & 50 & 2 \\
Landvolk & 28 & 44 & 6 & 6 & 3 \\
Sonstige & 24 & 38 & 6 & 7 & 4 \\
Nichtwähler & 15 & 22 & 6 & 7 & 25 \\
\hline \multicolumn{7}{|r|}{} & 2 & 4 & 100 & 7 & 100 \\
\hline
\end{tabular}

43 Zur Wirtschaftspartei vgl. M. Schumacher, Mittelstandsfront und Republik. Die Wirtschaftspartei - Reichspartei des deutschen Mittelstandes. 1919-1933, Düsseldorf 1972 . 
Lesebeispiel: 52 Prozent der DNVP-Wähler vom Mai 1928 befürworteten im Oktober 1929 das Volksbegehren und 65 Prozent der DNVP-Wähler vom Mai 1928 stimmten mit Ja beim Volksentscheid (linke Tabellenhälfte). Beim Volksbegehren 1929 stellten die ehemaligen DNVP-Wähler 57 Prozent, die NSDAP-Wähler 6 Prozent usw. der Befürworter. Abweichungen zu 100 sind rundungsbedingt. Abk.: RdM = Reichspartei des Deutschen Mittelstandes (Wirtschaftspartei).

c) Das konfessionell-sozioökonomische Profil der Befürworter des Plebiszits 1929

Auch für die wahlstatistische Analyse des Plebiszits 1929 wurden nach den gleichen Kriterien wie für 1926 zwölf konfessionell-sozioökonomische Subgruppen gebildet und die Mittelwerte des Zuspruchs bei Volksbegehren und Volksentscheid berechnet. Ergänzend wurden in der Grafik 4 die durchschnittlichen Anteile von DNVP und NSDAP als tragende politische Säulen des Plebiszits sowie die zusätzliche Mobilisierungsquote beim Volksentscheid dargestellt.

\section{Grafik 4: Prozentanteile beim Plebiszit 1929 nach konfessionell- sozioökonomischen Kontrastgruppen}

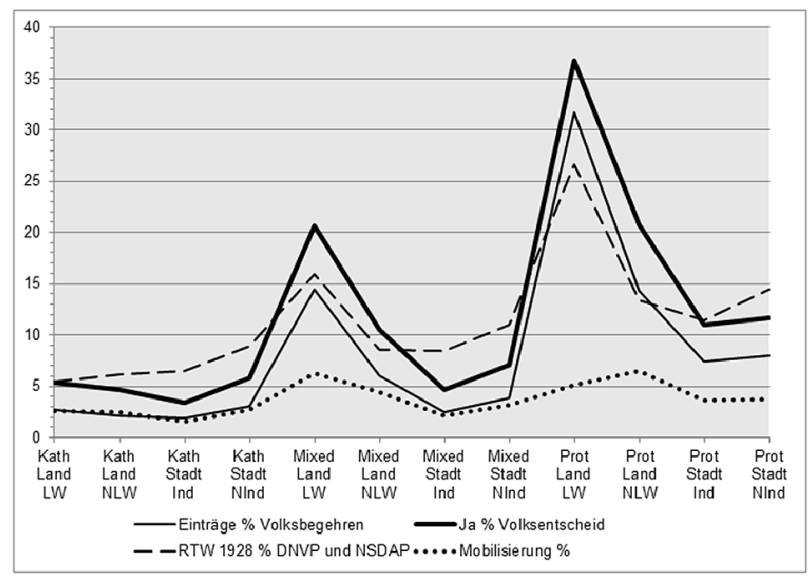

Die Hochburgen der Befürworter des Volksentscheids lagen in den evangelischen landwirtschaftlichen Kreisen, wo der Spitzenwert bei 37 Prozent beim Volksentscheid erzielt wurde. Dort ist der Abstand zwischen Zustimmung 
und DNVP-Anteilen 1928 am größten. Ziemlich erfolglos blieb das Plebiszit erneut im katholischen Bevölkerungssegment ${ }^{44}$. Das Zustimmungsprofil 1929 über die zwölf Kontexte verhält sich optisch genau gegenteilig zu dem des Plebiszits zur Fürstenenteignung 1926 und deckt sich weitgehend mit dem Erfolgsmuster der DNVP bei der vorausgegangenen Reichstagswahl 1928. Auch die zusätzlich mobilisierten Anteile beim Volksentscheid folgen andeutungsweise diesem Profil. Insgesamt zeigt sich, dass über die eigene Klientel hinaus kaum oder nur wenig neue Wählerschichten für das Plebiszit gewonnen wurden.

\section{d) Das Sozialprofil der Befürworter des Plebiszits 1929}

Eine Hochburgenanalyse mit einer Gegenüberstellung des Sozialprofils der Befürworter und Nichtbefürworter des Plebiszits verdeutlicht, welche soziale Milieuzusammensetzung für die Befürworter des Volksentscheids eher typisch war. 111 Städte, Kreise und Verwaltungsbezirke zählen zu den Hochburgen des Volksentscheids 1929, darunter an der Spitze - wie bereits erwähnt - der Kreis Rothenburg ob der Tauber (Wahlkreis 26, 76,0 Prozent bei 73,5 Prozent DNVPund NSDAP-Anteil), Kreis Uffenheim (Wahlkreis 26, 67,7 Prozent bei 58,9 Prozent), Kreis Ansbach Land (Wahlkreis 26, 63,4 Prozent bei 62,9 Prozent), Kreis Bremervoerde (Wahlkreis 15, 62,4 Prozent bei 10,1 Prozent plus 58,2 Prozent Landvolk) und Kreis Wildeshausen (Wahlkreis 14, 60,1 Prozent bei 27,4 Prozent). Die Hochburgen waren mit 93,7 Prozent gegenüber 62 Prozent in den Nichthochburgen eindeutig evangelischer geprägt. Die dominante Bedeutung der protestantisch-agrarischen Basis der Befürworter dokumentiert auch der Landwirtschaftsanteil in Höhe von 53,3 Prozent (versus 20,8 Prozent in den Nichthochburgen). Die Volksentscheide von 1926 und 1929 unterschieden sich hinsichtlich der Zusammensetzung der Hochburgen nach Wirtschaftsabteilungen recht stark voneinander: 1929 zählte in den Hochburgen der Befürworter des Young-Plebiszites 53,3 Prozent zum Landwirtschaftssektor (1926 in den Hochburgen: 5,7 Prozent), 20,5 Prozent dem Industrie- und Handwerkssektor (1926: 42,8 Prozent) und 16,9 Prozent zum Dienstleistungssektor (1926: 27,3 Prozent). Ähnlich unterschiedlich fiel die Aufgliederung nach sozialer Stellung aus: 20,9 Prozent betrug der Landarbeiteranteil in den 111 Hochburgen des Plebiszits 1929 (versus 5,4 Prozent in den restlichen Nichthochburgen), 31,2 Prozent der Anteil

44 Das zeigen auch die Korrelationen mit den Katholikenanteilen: Eintragsquote für das Volksbegehren -.489, Ja-Stimmen bei der Volksabstimmung -.533, Mobilisierung beim Volksentscheid -.325. 
der landwirtschaftlichen Selbständigen und Mithelfenden (versus 15,0 Prozent). Das Verhältnis der nichtlandwirtschaftlichen Selbständigen blieb mit 10,3 zu 13,9 Prozent relativ ausgeglichen. Unterdurchschnittlich repräsentiert blieben die nichtlandwirtschaftlichen Arbeiter mit 17,6 Prozent (gegenüber 37,5 Prozent) und die Angestellten und Beamten mit 8,8 Prozent (versus 16,9 Prozent) beim Vergleich der Hochburgen mit den Nichthochburgen. Diese Zahlen zeigen, dass der Volksentscheid 1929 seine soziale Basis überwiegend im wirtschaftlich rückständigen, agrarischen Deutschland fand und sich darin erheblich vom Volksentscheid 1926 unterschied.

Das Korrelationsmuster mit den Sozialstrukturvariablen belegt, dass die vorrangige Basis in den evangelischen ländlichen agrarischen Regionen lag. Stellvertretend dafür stehen die Landarbeiteranteile ${ }^{45}$. Das zeigt eine Kontrastgruppenanalyse mit den Faktoren Konfession und landwirtschaftlicher Arbeiteranteil, aufgeteilt in Quartile. Die ermittelten Schnittpunkte wurden auf die drei Konfessionsgruppen angewandt.

Ergebnis der so entstandenen Kontrastgruppen ist ein treppenartiges Muster der Zustimmung, das mit dem Landarbeiteranteil variiert, allerdings nur bei der evangelischen Landbevölkerung (vgl. Grafik 5 auf der folgenden Seite). Je höher der (protestantische) Landarbeiteranteil, desto erfolgreicher fielen Volksbegehren und Volksentscheid gegen den Young-Plan aus. Im untersten Quartil, das das Viertel der Kreise mit den niedrigsten protestantischen Landarbeiteranteilen repräsentiert, erreichte der Volksentscheid im Schnitt knapp elf Prozent Zustimmung, im Viertel mit den höchsten Anteilen waren es bereits 32,5 Prozent, doppelt so hoch wie im Reichsdurchschnitt.

Die Schätzungen zur sozialen Basis der Befürworter des Plebiszits deuten auf eine Veränderung ihrer Zusammensetzung zwischen Volksbegehren und Volksentscheid (Oktober und Dezember 1929) hin, gleichzeitig stieg der reichsweite Zuspruch von 10 auf 13,8 Prozent, also um gut ein Drittel. Überdurchschnittlich starke Unterstützung für Volksbegehren und Volksentscheid 1929 kam von den Landarbeitern, dem nichtlandwirtschaftlichen alten Mittelstand (Selbständige und Mithelfende) sowie von den Berufslosen. Beim Volksentscheid nahm der Zuspruch aus dem alten Mittelstand deutlich zu und verdoppelte sich fast. Ihr Anteil an den Ja-Stimmen stieg dadurch von einem Fünftel auf gut ein Viertel an, während gleichzeitig der Zuspruch unter den Landarbeitern von zwei auf ein Fünftel zurückging. Damit war die soziale

45 Die gewichteten Korrelationen zwischen Landarbeiteranteil und Plebiszit sind folgende: $+.707 ;+.636 ;-.010$ (Reihenfolge wie in Fn. 44). 
Basis der Befürworter des Volksentscheids anders noch als beim Volksbegehren etwas pluralistischer zusammengesetzt.

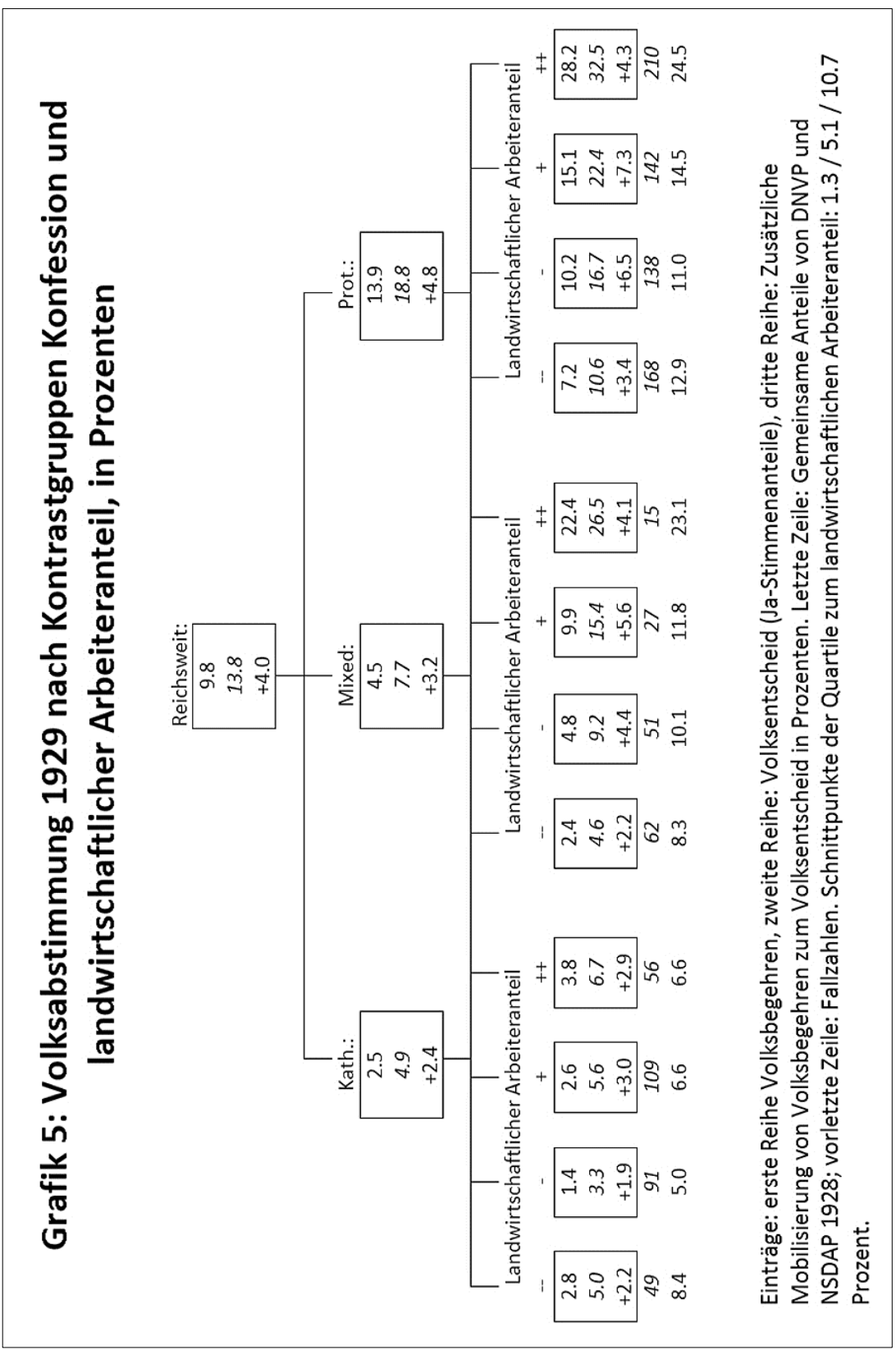


Tabelle 4: Geschätztes Abstimmungsverhalten nach sozialer Stellung bei Volksbegehren und Volksentscheid 1929 (Ja-Stimmenanteil) sowie die soziale Herkunft der Befürworter in gerundeten Prozenten

\begin{tabular}{|c|c|c|c|c|c|}
\hline & \multicolumn{2}{|c|}{$\begin{array}{c}\text { Zustimmung } \\
1929 \text { bei }\end{array}$} & \multicolumn{2}{|c|}{$\begin{array}{c}\text { Soziale Her- } \\
\text { kunft } 1929\end{array}$} & \multirow{2}{*}{$\begin{array}{c}\text { Berufs- } \\
\text { zählung } \\
1925\end{array}$} \\
\hline & VB & VE & VB & VE & \\
\hline LW Selbständige und Mithelfende & 4 & 11 & 6 & 12 & 16 \\
\hline NLW Selbständige und Mithelfende & 16 & 26 & 22 & 26 & 14 \\
\hline Angestellte und Beamte & 0 & 0 & 0 & 0 & 17 \\
\hline LW Arbeiter & 66 & 46 & 41 & 20 & 6 \\
\hline NLW Arbeiter & 1 & 5 & 3 & 12 & 36 \\
\hline \multirow[t]{2}{*}{ Berufslose } & 25 & 38 & 29 & 31 & 11 \\
\hline & & & 100 & 100 & 100 \\
\hline
\end{tabular}

Lesebeispiel: 4 Prozent der landwirtschaftlich Selbständigen und Mithelfenden befürworteten im Oktober 1929 das Volksbegehren, 11 Prozent stimmten mit Ja beim Volksentscheid (linke Tabellenhälfte). Beim Volksbegehren 1929 stellten die landwirtschaftlich Selbständigen und Mithelfenden 6 Prozent der Befürworter. Abweichungen zu 100 sind rundungsbedingt. Abk.: LW = Landwirtschaftlich; NLW = Nichtlandwirtschaftlich.

Setzt man diese Ergebnisse in Relation zu einer Schätzung der sozialen Herkunft der Wähler bei der vorangegangenen Reichstagswahl im Mai 1928, so sind beim Volksbegehren Parallelen zum Sozialprofil der DNVP-Wählerschaft unübersehbar ${ }^{46}$. Die DNVP wurde mit fast zwei Fünfteln am häufigsten von den Landarbeitern präferiert. 1928 stellten sie damit über ein Viertel der gesamten DNVP-Wählerschaft. Hinzu kamen die Berufslosen, die ebenfalls ein Viertel der deutschnationalen Wählerschaft ausmachten, während Selbständige und Mithelfende (insgesamt) deutlich weniger als ein Fünftel stellten (15 Prozent). Für die zusammengefasste Gruppe der sonstigen Parteien (darunter Landvolk und Wirtschaftspartei), aber auch für die NSDAP waren eher überdurchschnittlich hohe Anteile von Selbständigen und Mithelfenden charakteristisch, die schätzungsweise jeweils gut zwei Fünftel ausmachten. Diese Wählerprofile zeigen zusammengenommen mit dem oben skizzierten Sozialprofil der Befürworter des Volksentscheids im Dezember 1929 relativ gute Übereinstimmungen.

46 Aus Platzgründen kann an dieser Stelle nicht näher auf die Schätzergebnisse zur sozialen Basis der Parteien bei den beiden zeitlich ummantelnden Reichstagswahlen eingegangen werden. 


\section{Der Volksentscheid 1929 und der nationalsozialistische Durchbruch im September 1930}

Der Volksentscheid 1929 fiel in eine politisch und wirtschaftlich sehr bewegte Zeit. Der im Zuge des „schwarzen Freitags“ an der New Yorker Börse beginnende Rückgang der Industrieproduktion warf erste Schatten auf die deutsche Konjunktur. Mit dem Tod des DVP-Vorsitzenden und Außenministers Gustav Stresemann am 3. Oktober 1929 verlor die Weimarer Republik außerdem eine wichtige politische Integrationsfigur, deren Verlust für die Demokratie insbesondere im Folgejahr nicht hoch genug eingeschätzt werden kann.

Die zeitliche Nähe zur Septemberwahl 1930, bei der die NSDAP den reichsweiten Durchbruch erzielte, führte zu Diskussionen darüber, ob das Plebiszit 1929 den NSDAP-Aufstieg begünstigt hat ${ }^{47}$. Bbei dieser Reichstagswahl erzielte die NSDAP mit fast 14,9 Prozent Anteil (an den Wahlberechtigten) ein fulminantes Ergebnis gegenüber knapp zwei Prozent zwei Jahre zuvor.

Wenn die Befürworter überwiegend aus den Reihen der DNVP, Splitterparteien und NSDAP kamen, dann dürfte ein alleiniger Kausalzusammenhang zwischen Volksentscheidsvotum und NSDAP-Wahl eher unwahrscheinlich sein. Denn der Anstieg der Nationalsozialisten 1930 speiste sich, wie die wahlhistorische Forschung belegen konnte, aus mehreren Quellen: in erster Linie durch eine Mobilisierung ehemaliger Nichtwähler sowie stark zu Lasten von DVP und DNVP, die von 6,5 auf 3,7 bzw. von 10,6 auf 5,7 Prozent zurückgingen ${ }^{48}$.

In diesem Rahmen wurden auf der Basis der vorliegenden 1.118 Wahlund Sozialdaten für die Kreise und Städte bivariate Zusammenhänge zwischen Volksentscheid und dem Abschneiden der NSDAP berechnet sowie der Anteil der Plebiszitbefürworter an der späteren NSDAP-Wählerschaft geschätzt. Die Assoziationen sind auf der statistischen Beziehungsebene

47 O. Jung, „Plebiszitärer Durchbruch 1929? Zur Bedeutung von Volksbegehren und Volksentscheid gegen den Youngplan für die NSDAP“, in: GuG 15 (1989), S. 489510 sieht keinen direkten, sondern eher einen mittelbaren Zusammenhang.

48 Vgl. dazu die Analysen der Forschungsgruppe um den Politikwissenschaftler Falter, die dieser in Hitlers Wähler (Fn. 8) zusammengefasst hat. Vgl. zuvor dazu auch $T$. Childers, The Nazi Voter: The Social Foundations of Fascism in Germany, 19191933, Chapel Hill 1983, von dem der Begriff „,catch-all-party“ stammt, ferner auch D. Hänisch, Sozialstrukturelle Bestimmungsgründe des Wahlverhaltens in der Weimarer Republik 1924-1933, Duisburg 1982. 
recht deutlich: Zwischen den Anteilen der Ja-Stimmen 1929 und dem Abschneiden der NSDAP 1930 besteht ein starker Zusammenhang (+.568), ähnlich stark verhält es sich bei einer Korrelation mit dem prozentualen Gewinn der NSDAP 1930 gegenüber 1928 (+.541) bzw. der Verluste der DNVP 1930 $(-.562)$.

Wohin die Befürworter des Volksentscheids bei der anschließenden Reichstagswahl wanderten, zeigen die Schätzungen, die über die 1.118 Kreis- und Stadteinheiten, gegliedert in die konfessionell-sozioökonomischen Kontexte, errechnet wurden. Demnach gingen über zwei Fünftel der Plebiszitbefürworter zur NSDAP (43 Prozent), ein Fünftel zur DNVP (20 Prozent). Ein weiterer Adressat waren die sonstigen Parteien (14 Prozent) sowie SPD und Nichtwähler (je acht Prozent). Auch wenn diese Schätzungen nicht prozentgenau zutreffend sein dürften, verdeutlichen sie doch, dass die DNVP einen großen Teil ihrer Plebiszitbefürworter nicht mehr zurückgewinnen konnte und diese dann von der NSDAP erfolgreich aufgesogen wurden. Dieser Prozess ist vor allem auch im Zusammenhang mit dem beginnenden Zerfall der Landarbeiterbasis der DNVP zu sehen ${ }^{49}$. Insofern fungierte der Volksentscheid quasi als eine Art Zwischenwirt auf dem Weg der DNVP-Wähler hin zur NSDAP. Ähnlich, allerdings in deutlich kleinerem Ausmaß, verhielt es sich bei den übrigen Wanderungsbewegungen, von denen nur ein Teil zu ihrer Herkunftspartei wieder zurückfand. Insofern spiegelt sich im Plebiszit auch ein Stück der generellen Wählerbewegung von 1928 zu 1930 wider $^{50}$.

Weil die politische Herkunft der Nationalsozialisten eher pluralistisch zusammengesetzt war und sich aus mehreren Quellen rekrutierte, stellten die stark DNVP-gefärbten Plebiszitbefürworter von 1929 auch ,nur“ zwei Fünftel der NS-Wählerschaft von 1930. Die Mehrheit der neuen nationalsozialistischen Wähler hatte sich zuvor gar nicht am Volksentscheid beteiligt.

49 Vgl. W. Pyta, Dorfgemeinschaft und Parteipolitik 1918-1933. Die Verschränkung von Milieu und Parteien in den protestantischen Landgebieten Deutschlands in der Weimarer Republik, Düsseldorf 1996; ders., „Ländlich-evangelisches Milieu und Nationalsozialismus bis 1933“", in: H. Möller/A. Wirsching/W. Ziegler (Hrsg.), Nationalsozialismus in der Region, München 1996, S. 199-212.

50 Was auch für die Qualität dieser Schätzung spricht, denn im Fall der Splitterparteien (Christlich Sozialer Volksdienst, Konservative Volkspartei, Landbund etc.) hat bereits vor längerem die historische Wahlforschung nachgewiesen, dass nennenswerte Wählerwanderungen zur NSDAP von diesen insbesondere erst bei der Juliwahl 1932 erfolgten. 
Grafik 6: Die geschätzten Wählerzuflüsse zum Volksentscheid 1929 und die Wählerabwanderungen vom Volksentscheid zur Reichstagswahl 1930 (in Mio.)

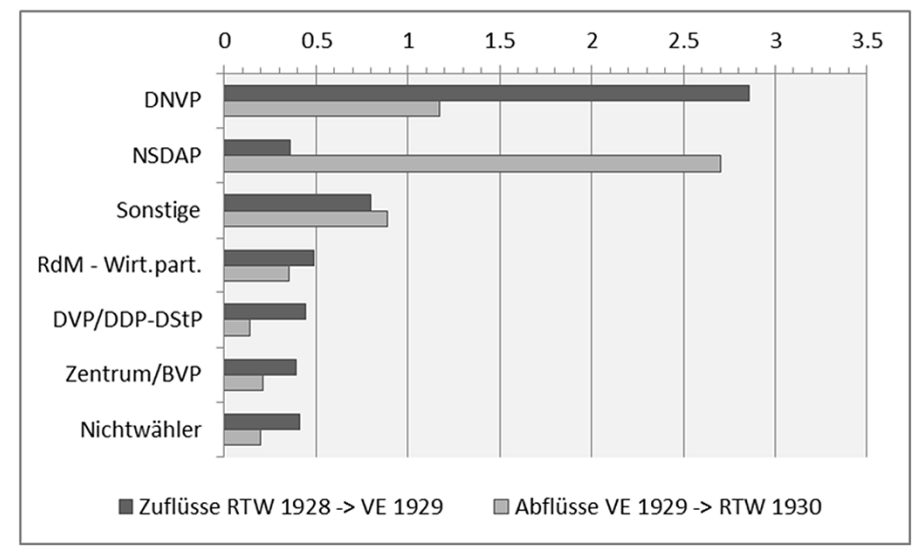

Anm.: Zu den „Sonstigen Parteien“ zählen Landvolk, Konservative Volkspartei, Christlich-Sozialer Volksdienst, Landbund und andere Klein- und Kleinstparteien. RdM - Wirt.part. = Reichspartei des deutschen Mittelstandes (Wirtschaftspartei). Lesebeispiel: Von der DNVP befürworteten 1928 knapp 2,9 Mio. Wähler den Volksentscheid 1929; knapp 1,2 Mio. der Befürworter des Volksentscheids wählten bei der darauffolgenden Reichstagswahl 1930 die DNVP

\section{5. $\quad$ Kurzresümee und Ausblick}

Die wahlstatistischen Analysen der beiden Plebiszite führen in vielerlei Hinsicht zu gegensätzlichen Befunden. War das Plebiszit zur Fürstenenteignung auf der Linken noch am ehesten von der politischen Unterstützung her eine Abstimmung mit Volksbewegungscharakter, wofür schon die Höhe der Unterstützung spricht, so blieb das Votum zur Ablehnung des Young-Plans weitgehend ein isoliertes Projekt der Deutschnationalen. Beiden Plebisziten war gemeinsam, dass sie kaum die katholische Bevölkerung auf dem Land und in den Städten erreichten.

Jede der beiden Abstimmungen stützte sich auf bestimmte soziale Segmente der Wählerschaft. Sie besaßen in dieser Hinsicht kaum eine schichtoder klassenübergreifende Basis. Das war natürlich in erster Linie der segmentierten Weimarer Parteienlandschaft geschuldet, in der lediglich die Zentrumspartei annähernd volksparteiähnlichen Charakter besaß - was aber nur für den katholischen Teil der Bevölkerung zutraf.

Die wahlstatistische Analyse der beiden Plebiszite hat darüber einige neue empirisch fundierte Erkenntnisse geliefert, von denen hier nur zwei herausgegriffen werden sollen. Zum einen wurde der Anteil der Zentrumswähler an den Befürwortern des Volksentscheids zur Enteignung des Fürstenvermögens in der Literatur offensichtlich überschätzt; andererseits wurde der Anteil 
an Wählern protestantischer Mittelschichtparteien wie der DVP unterschätzt. Insgesamt wirkte bei diesem Volksentscheid die konfessionelle Konfliktlinie weniger stark trennend als beim Plebiszit 1929. Bei diesem wurde bislang nicht zur Kenntnis genommen, dass sich zwischen Volksbegehren und Volksentscheid die soziale und politische Trägerbasis der Unterstützung veränderte. Deutlich gemacht werden konnte die Zwischenwirtfunktion des Volksentscheids für die DNVP-Wähler auf dem Weg hin zu den Nationalsozialisten. Dieser Volksentscheid selbst spielte für die Rekrutierung der nationalsozialistischen Wähler allerdings eher eine untergeordnete Rolle.

Hinsichtlich der Plebiszite in der Weimarer Republik bleibt für die zukünftige wahlhistorische Wahlforschung die eingehende Untersuchung der gescheiterten Volksentscheide zu den Landtagsauflösungen in der späten Weimarer Republik in Preußen im August 1931 und in Sachsen im April 1932 auf der Agenda. Hier ist beispielsweise noch gänzlich unerforscht, in welchem Ausmaß sich die KPD-Wählerschaft an den Volksentscheiden beteiligte. In welchem Ausmaß folgte sie ihrer Parteiführung und wie stark verweigerte die Anhängerschaft den Gang zur Wahlurne wegen der gleichzeitigen Unterstützung der Nationalsozialisten? Ebenso steht eine gründliche Untersuchung des reichsweiten Resistenzpotenzials bei den beiden Volksabstimmungen 1933 und 1934 sowie der Reichstags,,wahl“ November 1933 unter Hitler noch aus, wobei hier die diktatorischen Rahmenbedingungen und die besonders schwierigen Umstände für dissidentes Verhalten entsprechend zu berücksichtigen sind ${ }^{51}$. Die empirische Analyse auch dieser Plebiszite für das gesamte Deutsche Reich bleibt ein Desiderat.

51 Die einzigen Studien, die sich gründlicher mit den nationalsozialistisch inszenierten Abstimmungen beschäftigen, beziehen sich auf die über 200 Stimmbezirke der sächsischen Industriestadt Chemnitz, vgl. D. Hänisch, Das Wahl- und Abstimmungsverhalten in Chemnitz 1933 und 1934, in: Stadtarchiv Chemnitz (Hrsg.), Chemnitz in der NS-Zeit: Beiträge zur Stadtgeschichte 1933-1945, Leipzig 2008, S. 7-36; ferner auch zur Provinz Schleswig-Holstein: F. Omland, „Du wählst mi nich Hitler!“ Reichstagswahlen und Volksabstimmungen in Schleswig-Holstein, 1933-1938, Hamburg 2006 sowie ders., Plebiszite in der Zustimmungsdiktatur - Die nationalsozialistischen Volksabstimmungen 1933,1934 und 1938: das Beispiel SchleswigHolstein, in: L.P. Feld u.a. (Hrsg.), Jahrbuch für direkte Demokratie 2009, BadenBaden 2010, S. 131-159. 